ESAIM: M2AN

Vol. 40, No 4, 2006, pp. 705-734

DOI: $10.1051 / \mathrm{m} 2 \mathrm{an}: 2006031$
ESAIM: Mathematical Modelling and Numerical Analysis

www.edpsciences.org/m2an

\title{
VIBRATIONS OF A BEAM BETWEEN OBSTACLES. CONVERGENCE OF A FULLY DISCRETIZED APPROXIMATION
}

\author{
Yves Dumont $^{1}$ AND Laetitia PaOli ${ }^{2}$
}

\begin{abstract}
We consider mathematical models describing dynamics of an elastic beam which is clamped at its left end to a vibrating support and which can move freely at its right end between two rigid obstacles. We model the contact with Signorini's complementary conditions between the displacement and the shear stress. For this infinite dimensional contact problem, we propose a family of fully discretized approximations and their convergence is proved. Moreover some examples of implementation are presented. The results obtained here are also valid in the case of a beam oscillating between two longitudinal rigid obstacles.
\end{abstract}

Mathematics Subject Classification. 35L85, 65M12, 74H45.

Received: January 13, 2006. Revised: April 10, 2006.

\section{Description of the Problem}

We consider a beam which is clamped at its left end to a vibrating support and which can move freely between two rigid obstacles at its right end (see Fig. 1).

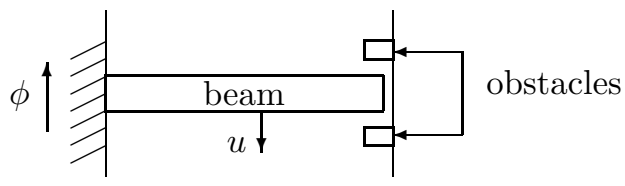

FiguRE 1. The physical setting.

The longitudinal axis of the beam coincide with the interval $[0, L]$ and we denote by $\tilde{u}(x, t),(x, t) \in(0, L) \times$ $(0, T)$ the vertical displacement of a point $x$ belonging to this axis. We assume that the material is elastic and the motion is planar. We denote by $\tilde{\sigma}$ the shear stress given by

$$
\tilde{\sigma}(x, t)=-k^{2} \tilde{u}_{x x x}, \quad k^{2}=\frac{E I}{\rho S}
$$

Keywords and phrases. Dynamics with impact, Signorini's conditions, space and time discretization, convergence.

${ }^{1}$ IREMIA, Université de La Réunion, 15 avenue R. Cassin, 97715 Saint-Denis Messag. 9, France. Yves.Dumont@univ-reunion.fr

2 LaMUSE, Université de St-Étienne, 23 rue P. Michelon, 42023 St-Étienne Cedex 2, France.

laetitia.paoli@univ-st-etienne.fr 
where $\rho$ and $E$ are the density and the Young's modulus of the material and $S$ and $I$ are respectively the surface and the moment of the cross section of the beam. Then, under the assumption of small displacements, the motion is described by the following partial differential equation

$$
\tilde{u}_{t t}-\tilde{\sigma}_{x}=\tilde{f}
$$

where $\tilde{f}$ is the density of external forces.

The beam is clamped at its left end so

$$
\tilde{u}(0, t)=\phi(t), \quad \tilde{u}_{x}(0, t)=0
$$

where $\phi$ describes the motion of the vibrating support. At its right end the beam can move freely between two obstacles, so we have

$$
g_{1} \leq \tilde{u}(L, t) \leq g_{2}, \quad \tilde{u}_{x x}(L, t)=0
$$

and we assume that $g_{1}<0<g_{2}$. When the beam hits one of the two obstacles, the stress is in the opposite direction of the displacement and we obtain the following Signorini's conditions

$$
\begin{cases}\tilde{\sigma}(L, t) \geq 0 & \text { if } \tilde{u}(L, t)=g_{1} \\ \tilde{\sigma}(L, t) \leq 0 & \text { if } \tilde{u}(L, t)=g_{2} \\ \tilde{\sigma}(L, t)=0 & \text { if } g_{1}<\tilde{u}(L, t)<g_{2}\end{cases}
$$

These relations can be rewritten as follows

$$
-\tilde{\sigma}(L, t) \in \partial \psi_{\left[g_{1}, g_{2}\right]}(\tilde{u}(L, t))
$$

where $\psi_{\left[g_{1}, g_{2}\right]}$ is the indicator function of the interval $\left[g_{1}, g_{2}\right]$ and $\partial \psi_{\left[g_{1}, g_{2}\right]}$ is its subdifferential [18].

In order to deal with homogeneous boundary conditions at $x=0$, we consider a new unknown function $u$ defined by

$$
u(x, t)=\tilde{u}(x, t)-h(x) \phi(t)
$$

with

$$
h(x)=1-2\left(\frac{x}{L}\right)^{2}+\frac{4}{3}\left(\frac{x}{L}\right)^{3}-\frac{1}{3}\left(\frac{x}{L}\right)^{4}
$$

and we let

$$
\sigma(x, t)=-k^{2} u_{x x x}(x, t)
$$

The mechanical problem is now described by the system

$$
\left\{\begin{array}{l}
u_{t t}+k^{2} u_{x x x x}=f \quad \text { in }(0, L) \times(0, T) \\
u(0, \cdot)=u_{x}(0, \cdot)=u_{x x}(L, \cdot)=0 \quad \text { in }(0, T) \\
u(L, \cdot) \in\left[g_{1}, g_{2}\right], u_{x x x}(L, \cdot) \in \partial \psi_{\left[g_{1}, g_{2}\right]}(u(L, \cdot)) \quad \text { in }(0, T)
\end{array}\right.
$$

with $f(x, t)=\tilde{f}(x, t)-h(x) \phi^{\prime \prime}(t)-k^{2} h^{(4)}(x) \phi(t)$ for all $(x, t) \in(0, L) \times(0, T)$. We complete the model with the initial conditions

$$
u(\cdot, 0)=u_{0}, \quad u_{t}(\cdot, 0)=v_{0} \quad \text { in }(0, L)
$$


As usual in mechanical problems with unilateral constraints we cannot expect smooth solutions since the velocities of the right extremity of the beam may be discontinuous at impacts. Indeed, assume that the beam hits one of the obstacles at $t_{0} \in(0, T)$, i.e. $u\left(L, t_{0}\right)=g_{1}$ and $u(L, t) \in\left(g_{1}, g_{2}\right)$ for all $t \in\left(t_{0}-\epsilon, t_{0}+\epsilon\right) \backslash\left\{t_{0}\right\}$ $(\epsilon>0)$ for instance. Then the ratio $\frac{u(L, t)-u\left(L, t_{0}\right)}{t-t_{0}}$ is non positive on $\left(t_{0}-\epsilon, t_{0}\right)$ while it is non negative on $\left(t_{0}, t_{0}+\epsilon\right)$. So we consider a variational formulation of the problem. For this purpose we introduce the following functional spaces

$$
\begin{gathered}
H=L^{2}(0, L), \quad V=\left\{w \in H^{2}(0, L) ; w(0)=w_{x}(0)=0\right\}, \\
\mathcal{H}=\left\{w \in L^{2}(0, T ; V) ; w_{t} \in L^{2}(0, T ; H)\right\},
\end{gathered}
$$

and we expect solutions $u \in \mathcal{H} \cap L^{2}(0, T ; K)$, where $K$ is the convex set

$$
K=\left\{w \in V ; g_{1} \leq w(L) \leq g_{2}\right\}
$$

We denote by $(.,$.$) and |$.$| the canonical scalar product and norm of H$. Let $a$ be the following bilinear form

$$
a(u, v)=\int_{0}^{L} k^{2} u_{x x} v_{x x} \mathrm{~d} x \quad \forall(u, v) \in V^{2} .
$$

We may observe that $a$ defines a scalar product on $V$ and the associated norm, denoted $\|\cdot\|_{V}$, is equivalent to the canonical norm of $H^{2}(0, L)$ on $V$. The weak formulation of the problem is then given by the following variational inequality

$$
(P)\left\{\begin{array}{l}
-\int_{0}^{T}\left(u_{t}(\cdot, t), w_{t}(\cdot, t)-u_{t}(\cdot, t)\right) \mathrm{d} t+\int_{0}^{T} a(u(\cdot, t), w(\cdot, t)-u(\cdot, t)) \\
\geq\left(v_{0}, w(\cdot, 0)-u_{0}\right)+\int_{0}^{T}(f(\cdot, t), w(\cdot, t)-u(\cdot, t)) \mathrm{d} t \\
\forall w \in \mathcal{H} \cap L^{2}(0, T ; K) \text { such that } w(\cdot, T)=u(\cdot, T) .
\end{array}\right.
$$

For this problem an existence result has been obtained by K. Kuttler and M. Shillor by using a penalty method.

Theorem 1.1. [8] Assume that $f \in L^{2}(0, T ; H), u_{0} \in K, v_{0} \in H$. Then there exists $u \in \mathcal{H} \cap L^{2}(0, T ; K)$ such that problem $(P)$ is satisfied and $u(\cdot, 0)=u_{0}$.

It should be noted that, as far as we know, uniqueness remains an open question.

For the computation of approximate solutions, the penalty method which is introduced as a theoretical tool to obtain existence in [8] could appear as an interesting technique: the Signorini's conditions are replaced by a normal compliance law

$$
\sigma(L, t)=-\frac{1}{\varepsilon}\left[\max \left(u(L, t)-g_{2}, 0\right)-\max \left(g_{1}-u(L, t), 0\right)\right], \quad \varepsilon \ll 1
$$

which leads to a system of partial differential equations depending on the penalty parameter $\varepsilon$ and a solution of $(\mathrm{P})$ is obtained as the limit of a converging subsequence of the penalized problems in $W=\{w \in$

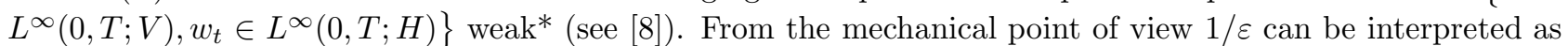
the stiffness of the obstacles which are not assumed to be perfectly rigid anymore. From a numerical point of view, for small values of $\varepsilon$, we have to deal with a very stiff partial differential equation. By a discretization in space, the problem reduces to a stiff second order differential equation of the same type as the one studied in [14]. It has been proved in [14] that the length of the time interval during which the system does not satisfy the constraint is of order $\mathcal{O}(\sqrt{\varepsilon})$. It follows that we should choose a time step smaller than $\mathcal{O}(\sqrt{\varepsilon})$, which is 
expensive (see [2] for a comparison of several schemes applied to the normal compliance approximate problem; see also [1] for more comments on the penalty method). Moreover the dynamics of the system may be complex (see [10] for a periodic forcing) and the approximate motion could be quite sensitive with respect to the value of $\varepsilon$ (see [15] for an example in the case of a simplified model of vibrations, see also [1]).

In order to avoid these difficulties, we propose to deal directly with the unilateral boundary condition by solving a complete discretization, in both time and space, of the variational inequality $(\mathrm{P})$.

From now on we will consider the more general case of a convex set $K$ given by

$$
K=\left\{w \in V ; g_{1}(x) \leq w(x) \leq g_{2}(x) \quad \forall x \in[0, L]\right\}
$$

where $g_{1}, g_{2}$ are two mappings from $[0, L]$ to $\overline{\mathbb{R}}$ such that there exists $g>0$ such that

$$
g_{1}(x) \leq-g<0<g \leq g_{2}(x) \quad \forall x \in[0, L] .
$$

We should notice that this framework includes the case of punctual obstacles since $g_{1}(x)$ and $g_{2}(x)$ may be equal to $-\infty$ and $+\infty$ respectively, as well as the case of two longitudinal rigid obstacles (see Fig. 2).

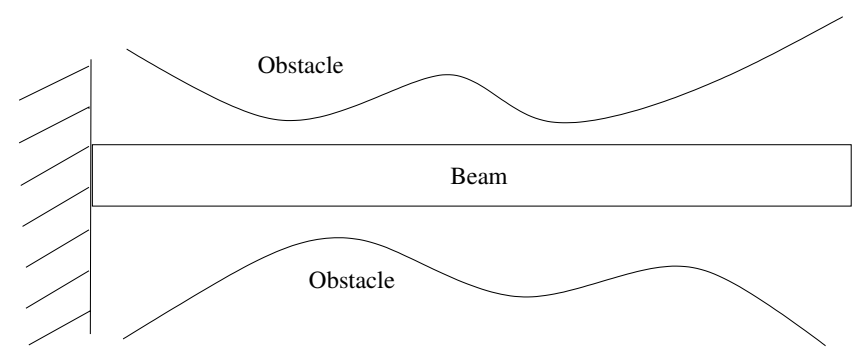

Figure 2. Beam between longitudinal rigid obstacles.

The paper is organized as follows: in the next section we introduce the fully discretized approximation of the problem, then in Section 3, we prove its stability and convergence and finally, in Section 4, we present some examples of implementation.

We may observe that the convergence result yields also an existence result for the more general case that we consider here. Moreover, let us outline that there exist very few convergence results for fully discretized approximations of variational inequalities describing the dynamics of elastic bodies submitted to perfect unilateral constraints. As far as we know, only the case of longitudinal vibrations of a rod, whose motion is limited by a rigid obstacle at one end, has been considered (see [19]).

\section{Discretization}

Let us first consider the case of two rigid obstacles at the right end of the beam (see Fig. 1), i.e. $K$ is defined by (1). We can derive a semi-discretization in space of the problem by applying a P3 finite element approximation. So we consider a partition of the interval $[0, L]$ into $J$ subintervals of length $h$, i.e. $x_{0}=0$, $x_{i}=i h, \ldots, x_{J}=L$. We use the well-known Hermite piecewise cubics as basis functions. More precisely, at each node $x_{i}$, we associate two Hermite piecewise cubics $\varphi_{2 i-1}$ and $\varphi_{2 i}$ defined by

$$
\begin{aligned}
& \varphi_{2 i-1} \in P_{3}, \varphi_{2 i-1}\left(x_{j}\right)=\delta_{i j} \text { and } \varphi_{2 i-1}^{\prime}\left(x_{j}\right)=0 \quad \text { for } 1 \leq j \leq J \\
& \varphi_{2 i} \in P_{3}, \varphi_{2 i}^{\prime}\left(x_{j}\right)=\delta_{i j} \text { and } \varphi_{2 i}\left(x_{j}\right)=0 \quad \text { for } 1 \leq j \leq J
\end{aligned}
$$


We introduce the following finite dimensional subspace

$$
V_{h}=\operatorname{span}\left\{\varphi_{1}, \varphi_{2}, \ldots, \varphi_{2 J-1}, \varphi_{2 J}\right\} \subset V .
$$

Thus, for all $u_{h} \in V_{h}$ we have

$$
u_{h}=\sum_{i=1}^{2 J} \bar{u}_{i} \varphi_{i}
$$

where

$$
\bar{u}_{2 i-1}=u_{h}\left(x_{i}\right), \quad \bar{u}_{2 i}=u_{h}^{\prime}\left(x_{i}\right) \quad \forall i \in\{1, \ldots, J\} .
$$

Then, the semi-discretization in space of the unconstrained problem leads to an ordinary differential equation in $\mathbb{R}^{2 J}$

$$
\mathcal{M} \ddot{\bar{u}}+\mathcal{S} \bar{u}=\mathcal{F}
$$

where $\mathcal{M}$ and $\mathcal{S}$ are respectively the global mass and stiffness matrices and

$$
\mathcal{F}_{i}=\left(f, \varphi_{i}\right) \quad \forall i \in\{1, \ldots, 2 J\} .
$$

If we take into account the unilateral constraint we have also the condition

$$
u_{h}(L, t)=\sum_{i=1}^{2 J} \bar{u}_{i}(t) \varphi_{i}(L)=\bar{u}_{2 J-1}(t) \in\left[g_{1}, g_{2}\right] \quad \forall t \in(0, T)
$$

which is equivalent to the condition $u_{h} \in V_{h} \cap K=K_{h}$.

Hence, we have to solve the following differential inclusion

$$
\mathcal{M} \ddot{\bar{u}}+\mathcal{S} \bar{u}+\partial \psi_{\bar{K}_{h}}(\bar{u}) \ni \mathcal{F}
$$

where $\bar{K}_{h}=\mathbb{R}^{2 J-2} \times\left[g_{1}, g_{2}\right] \times \mathbb{R}$.

Here $\bar{u}$ can be interpreted as the representative point of a system of rigid bodies with $2 J$ degrees of freedom, which dynamics is described by the measure differential inclusion (4). In order to obtain a complete discretization of the problem, we propose to apply to (4) a time-stepping scheme inspired by [11] (see also [16] or [13]), i.e.

$$
\mathcal{M} \frac{\bar{u}^{n+1}-2 \bar{u}^{n}+\bar{u}^{n-1}}{\Delta t^{2}}+\partial \psi_{\bar{K}_{h}}\left(\bar{u}^{n+1}\right) \ni \mathcal{G}^{n}
$$

where $\mathcal{G}^{n}$ is an approximation of $\mathcal{F}-\mathcal{S} \bar{u}$ at time $t_{n}$. Since second order Newmark's algorithms, of parameters $\gamma=1 / 2$ and $\beta \in[0,1 / 2]$ [7], (and derivatives) are extensively used in mechanics and in engineering, we choose

$$
\mathcal{G}^{n}=\beta\left(\mathcal{F}^{n+1}-\mathcal{S} \bar{u}^{n+1}\right)+(1-2 \beta)\left(\mathcal{F}^{n}-\mathcal{S} \bar{u}^{n}\right)+\beta\left(\mathcal{F}^{n-1}-\mathcal{S} \bar{u}^{n-1}\right)
$$

with

$$
\mathcal{F}_{i}^{n}=\frac{1}{\Delta t} \int_{n \Delta t}^{(n+1) \Delta t}\left(f(\cdot, s), \varphi_{i}\right) \mathrm{d} s \quad \forall i \in\{1, \ldots, 2 J\} .
$$


Recalling that $\partial \psi_{\bar{K}_{h}}\left(\bar{u}^{n+1}\right)$ is defined by

$$
\partial \psi_{\bar{K}_{h}}\left(\bar{u}^{n+1}\right)= \begin{cases}\left\{x \in \mathbb{R}^{2 J} ;\left(x, \bar{w}-\bar{u}^{n+1}\right) \leq 0 \forall \bar{w} \in \bar{K}_{h}\right\} & \text { if } \bar{u}^{n+1} \in \bar{K}_{h}, \\ \emptyset & \text { otherwise }\end{cases}
$$

we get

$$
\left\{\begin{array}{l}
\text { find } \bar{u}^{n+1} \in \bar{K}_{h}=\mathbb{R}^{2 J-2} \times\left[g_{1}, g_{2}\right] \times \mathbb{R} \text { such that, for all } \bar{w} \in \bar{K}_{h} \\
\left(\mathcal{M}\left(\frac{\bar{u}^{n+1}-2 \bar{u}^{n}+\bar{u}^{n-1}}{\Delta t^{2}}\right), \bar{w}-\bar{u}^{n+1}\right) \\
+\left(\mathcal{S}\left(\beta \bar{u}^{n+1}+(1-2 \beta) \bar{u}^{n}+\beta \bar{u}^{n-1}\right), \bar{w}-\bar{u}^{n+1}\right) \\
\geq\left(\beta \mathcal{F}^{n+1}+(1-2 \beta) \mathcal{F}^{n}+\beta \mathcal{F}^{n-1}, \bar{w}-\bar{u}^{n+1}\right) .
\end{array}\right.
$$

This problem can be rewritten in a more "variational" form as

$$
\left(P_{h \beta}^{n+1}\right)\left\{\begin{array}{l}
\text { find } u_{h}^{n+1} \in K_{h}=V_{h} \cap K \text { such that } \\
\left(\frac{u_{h}^{n+1}-2 u_{h}^{n}+u_{h}^{n-1}}{\Delta t^{2}}, w_{h}-u_{h}^{n+1}\right) \\
+a\left(\beta u_{h}^{n+1}+(1-2 \beta) u_{h}^{n}+\beta u_{h}^{n-1}, w_{h}-u_{h}^{n+1}\right) \\
\geq\left(\beta f^{n+1}+(1-2 \beta) f^{n}+\beta f^{n-1}, w_{h}-u_{h}^{n+1}\right) \quad \forall w_{h} \in K_{h}
\end{array}\right.
$$

with

$$
f^{n}=\frac{1}{\Delta t} \int_{n \Delta t}^{(n+1) \Delta t} f(\cdot, s) \mathrm{d} s .
$$

We may observe that this general formulation of the discretized problem allows us to consider other space discretizations derived from other approximations of $V$ (like spline approximations or other Galerkin approximations for instance) as well as the case of longitudinal rigid obstacles.

The dynamical behaviour of mechanical systems submitted to perfect unilateral constraints is often very complex: impacts accumulation, sensitivity to initial data and even chaos may occur (see [10], [20] or [6]). In this context, it seems almost impossible to determine error estimates for the proposed numerical method. Moreover, the convergence order is not an essential point since any prescribed accuracy will be lost in finite time. Thus we will prove only a convergence result. Nevertheless, we know that the time-stepping scheme that we apply to the semi-discretized problem is at most of order 1 ([12], see also [9]). So we may infer that the approximate solutions are at most of order 1 in time.

Let us assume from now on that the convex set $K$ is given by (2)-(3) and that the assumptions of Theorem 1.1 hold, i.e. $f \in L^{2}(0, T ; H), u_{0} \in K$ and $v_{0} \in H$.

For all $h \in \mathbb{R}_{+}^{*}$ we consider a finite dimensional subspace $V_{h}$ of $V$ such that, for all $v \in V$, there exists a sequence $\left(v_{h}\right)_{h>0}$ such that

$$
\left\|v_{h}-v\right\|_{V} \rightarrow_{h \rightarrow 0} 0, \quad v_{h} \in V_{h} \quad \forall h>0
$$

and we denote by $Q_{h}$ the projection onto $V_{h}$ with respect to the scalar product defined by $a$ on $V$. The compact embedding of $V$ into $H^{1}(0, L)$ implies that there exists a sequence $\left(\gamma_{h}\right)_{h>0}$ such that

$$
\forall w \in V \quad\left\|Q_{h}(w)-w\right\|_{H^{1}(0, L)} \leq \gamma_{h}\|w\|_{V}, \quad \lim _{h \rightarrow 0} \gamma_{h}=0 .
$$

For all $h>0$ we define $K_{h}=V_{h} \cap K$. 
Let $N \in \mathbb{N}^{*}$ and $\Delta t=T / N$. We propose the following family of discretizations of problem (P): For all $n \in\{1, \ldots, N-1\}$, find $u_{h}^{n+1} \in K_{h}$ such that

$$
\left(P_{h \beta}^{n+1}\right)\left\{\begin{array}{l}
\left(\frac{u_{h}^{n+1}-2 u_{h}^{n}+u_{h}^{n-1}}{\Delta t^{2}}, w_{h}-u_{h}^{n+1}\right) \\
+a\left(\beta u_{h}^{n+1}+(1-2 \beta) u_{h}^{n}+\beta u_{h}^{n-1}, w_{h}-u_{h}^{n+1}\right) \\
\geq\left(\beta f^{n+1}+(1-2 \beta) f^{n}+\beta f^{n-1}, w_{h}-u_{h}^{n+1}\right) \quad \forall w_{h} \in K_{h}
\end{array}\right.
$$

with

$$
f^{n}=\frac{1}{\Delta t} \int_{n \Delta t}^{(n+1) \Delta t} f(\cdot, s) \mathrm{d} s
$$

where $\beta$ is a parameter belonging to $[0,1 / 2]$. We choose $u_{h}^{0}$ and $u_{h}^{1}$ in $K_{h}$ such that $\left(\left\|u_{h}^{1}\right\|_{V}\right)_{h>0}$ remains bounded and

$$
\lim _{h \rightarrow 0, \Delta t \rightarrow 0}\left\|u_{h}^{0}-u_{0}\right\|_{V}+\left|\frac{u_{h}^{1}-u_{h}^{0}}{\Delta t}-v_{0}\right|=0 .
$$

We may observe that problem $\left(P_{h \beta}^{n+1}\right)(\beta \in[0,1 / 2])$ can be rewritten as

$$
\left\{\begin{array}{l}
\text { find } u_{h}^{n+1} \in K_{h} \text { such that } \\
a_{n \beta}\left(u_{h}^{n+1}, w_{h}-u_{h}^{n+1}\right) \geq L_{n \beta}\left(w_{h}-u_{h}^{n+1}\right) \quad \forall w_{h} \in K_{h}
\end{array}\right.
$$

with

$$
\begin{aligned}
& a_{n \beta}\left(u_{h}, v_{h}\right)=\left(u_{h}, v_{h}\right)+\Delta t^{2} \beta a\left(u_{h}, v_{h}\right) \\
& L_{n \beta}\left(v_{h}\right)=\Delta t^{2}\left(\beta f^{n+1}+(1-2 \beta) f^{n}+\beta f^{n-1}, v_{h}\right)+\left(2 u_{h}^{n}-u_{h}^{n-1}, v_{h}\right) \\
& \quad-\Delta t^{2} a\left((1-2 \beta) u_{h}^{n}+\beta u_{h}^{n-1}, v_{h}\right)
\end{aligned}
$$

for all $\left(u_{h}, v_{h}\right) \in V_{h}^{2}$.

By induction on $n$, we obtain that $L_{n \beta}$ is linear and continuous on $V_{h}$, and it is obvious that $a_{n \beta}$ is bilinear, symmetric, continuous and coercive on $V_{h}$. Thus the existence and uniqueness of $u_{h}^{n+1}$ follows from standard results on variational inequalities.

Remark 2.1. If $K=V$, i.e. $g_{1}(x)=-\infty, g_{2}(x)=+\infty$ for all $x \in[0, L]$, problem $\left(P_{h \beta}^{n+1}\right)$ reduces to

$$
\left\{\begin{array}{l}
\text { find } u_{h}^{n+1} \in V_{h} \text { such that } \\
\left(\frac{u_{h}^{n+1}-2 u_{h}^{n}+u_{h}^{n-1}}{\Delta t^{2}}, w_{h}\right)+a\left(\beta u_{h}^{n+1}+(1-2 \beta) u_{h}^{n}+\beta u_{h}^{n-1}, w_{h}\right) \\
=\left(\beta f^{n+1}+(1-2 \beta) f^{n}+\beta f^{n-1}, w_{h}\right) \quad \forall w_{h} \in V_{h}
\end{array}\right.
$$

which is simply the second order Newmark's scheme of parameters $\gamma=1 / 2$ and $\beta \in[0,1 / 2]$. In this case, the more usual choice of $\beta$ is $\beta \in[1 / 4,1 / 2]$, which corresponds to a necessary condition of stability for the discretization of the unconstrained linear problem. Because of the unilateral constraint, our problem becomes non linear and thus the stability properties are modified. However, we will show in the next section that unconditional stability is achieved for a particular value of $\beta$ when we deal with the constrained problem.

\section{Convergence}

Since the proposed discretizations are inspired by Newmark's method the stability of which depends on the value of $\beta$, we may expect the same kind of result for $\left(P_{h \beta}^{n+1}\right)$. More precisely, for $\beta \in[0,1 / 2)$ we obtain the following conditional stability property: 
Proposition 3.1. Let $\beta \in[0,1 / 2), h>0$ and $\kappa_{h}$ be defined by

$$
\kappa_{h}=\sup _{u_{h} \in V_{h} \backslash\{0\}} \frac{a\left(u_{h}, u_{h}\right)}{\left|u_{h}\right|^{2}} .
$$

Let $\alpha \in(0,1)$ and $N_{h} \in \mathbb{N}^{*}$ be such that

$$
\frac{T}{N_{h}}<\min \left(\sqrt{\frac{2(1-\alpha)}{\kappa_{h}(1-2 \beta)}}, \alpha\right) .
$$

Then there exists a constant depending only on the data, $C\left(f, u_{0}, v_{0}\right)$, such that for all $h>0$ and for all $N \geq N_{h}$ (i.e. $\Delta t \leq \Delta t_{h}=\frac{T}{N_{h}}$ )

$$
\alpha\left|\frac{u_{h}^{n+1}-u_{h}^{n}}{\Delta t}\right|^{2}+\frac{1}{2} a\left(u_{h}^{n}, u_{h}^{n}\right)+\frac{1}{2} a\left(u_{h}^{n+1}, u_{h}^{n+1}\right) \leq C\left(f, u_{0}, v_{0}\right)
$$

for all $n \in\{1, \ldots, N-1\}$, where $\left(u_{h}^{n+1}\right)_{1 \leq n \leq N-1}$ are the solutions of problems $\left(P_{h \beta}^{n+1}\right)_{1 \leq n \leq N-1}$.

Remark 3.2. An estimate of $\kappa_{h}$ in the case of a P3 finite element space discretization is given in the Appendix.

Proof. Let $n \in\{1, \ldots, N-1\}$ and choose $w_{h}=u_{h}^{n-1}$ as a test-function in $\left(P_{h \beta}^{n+1}\right)$. We get

$$
\left\{\begin{array}{l}
\left(\frac{u_{h}^{n+1}-2 u_{h}^{n}+u_{h}^{n-1}}{\Delta t^{2}}, u_{h}^{n-1}-u_{h}^{n+1}\right) \\
+a\left(\beta u_{h}^{n+1}+(1-2 \beta) u_{h}^{n}+\beta u_{h}^{n-1}, u_{h}^{n-1}-u_{h}^{n+1}\right) \geq\left(g^{n}, u_{h}^{n-1}-u_{h}^{n+1}\right)
\end{array}\right.
$$

where

$$
g^{n}=\beta f^{n+1}+(1-2 \beta) f^{n}+\beta f^{n-1} .
$$

The first two terms can be rewritten as follows:

$$
\left(\frac{u_{h}^{n+1}-2 u_{h}^{n}+u_{h}^{n-1}}{\Delta t^{2}}, u_{h}^{n-1}-u_{h}^{n+1}\right)=\left|\frac{u_{h}^{n-1}-u_{h}^{n}}{\Delta t}\right|^{2}-\left|\frac{u_{h}^{n+1}-u_{h}^{n}}{\Delta t}\right|^{2},
$$

and

$$
\begin{aligned}
& a\left(\beta u_{h}^{n+1}+(1-2 \beta) u_{h}^{n}+\beta u_{h}^{n-1}, u_{h}^{n-1}-u_{h}^{n+1}\right)=(1-2 \beta) a\left(u_{h}^{n-1}, u_{h}^{n}\right) \\
& +\beta a\left(u_{h}^{n-1}, u_{h}^{n-1}\right)-(1-2 \beta) a\left(u_{h}^{n}, u_{h}^{n+1}\right)-\beta a\left(u_{h}^{n+1}, u_{h}^{n+1}\right) .
\end{aligned}
$$

Hence, for all $n \in\{1, \ldots, N-1\}$, we have

$$
\begin{aligned}
& \left|\frac{u_{h}^{n+1}-u_{h}^{n}}{\Delta t}\right|^{2}+(1-2 \beta) a\left(u_{h}^{n}, u_{h}^{n+1}\right)+\beta a\left(u_{h}^{n+1}, u_{h}^{n+1}\right) \\
& \quad \leq\left|\frac{u_{h}^{n}-u_{h}^{n-1}}{\Delta t}\right|^{2}+(1-2 \beta) a\left(u_{h}^{n-1}, u_{h}^{n}\right)+\beta a\left(u_{h}^{n-1}, u_{h}^{n-1}\right)+\left(g^{n}, u_{h}^{n+1}-u_{h}^{n-1}\right)
\end{aligned}
$$


and with a discrete integration

$$
\begin{aligned}
&\left|\frac{u_{h}^{n+1}-u_{h}^{n}}{\Delta t}\right|^{2}+(1-2 \beta) a\left(u_{h}^{n}, u_{h}^{n+1}\right)+\beta a\left(u_{h}^{n+1}, u_{h}^{n+1}\right)+\beta a\left(u_{h}^{n}, u_{h}^{n}\right) \\
& \leq\left|\frac{u_{h}^{1}-u_{h}^{0}}{\Delta t}\right|^{2}+(1-2 \beta) a\left(u_{h}^{0}, u_{h}^{1}\right)+\beta a\left(u_{h}^{1}, u_{h}^{1}\right)+\beta a\left(u_{h}^{0}, u_{h}^{0}\right)+\sum_{p=1}^{n}\left(g^{p}, u_{h}^{p+1}-u_{h}^{p-1}\right)
\end{aligned}
$$

Using the same techniques as in [19], we define

$$
R\left(u_{h}, v_{h}\right)=(1-2 \beta) a\left(u_{h}, v_{h}\right)+\left|\frac{u_{h}-v_{h}}{\Delta t}\right|^{2} \quad \forall\left(u_{h}, v_{h}\right) \in V_{h}^{2} .
$$

We observe that

$$
\begin{aligned}
& R\left(u_{h}, v_{h}\right)=\frac{1-2 \beta}{2}\left(a\left(u_{h}, u_{h}\right)\right.\left.+a\left(v_{h}, v_{h}\right)-a\left(u_{h}-v_{h}, u_{h}-v_{h}\right)\right) \\
&+\left|\frac{u_{h}-v_{h}}{\Delta t}\right|^{2} \geq \frac{1-2 \beta}{2}\left(a\left(u_{h}, u_{h}\right)+a\left(v_{h}, v_{h}\right)\right)+\left(1-\kappa_{h} \Delta t^{2} \frac{1-2 \beta}{2}\right)\left|\frac{u_{h}-v_{h}}{\Delta t}\right|^{2}
\end{aligned}
$$

and with assumption (6), we infer that

$$
R\left(u_{h}, v_{h}\right) \geq \frac{1-2 \beta}{2}\left(a\left(u_{h}, u_{h}\right)+a\left(v_{h}, v_{h}\right)\right)+\alpha\left|\frac{u_{h}-v_{h}}{\Delta t}\right|^{2} \quad \forall\left(u_{h}, v_{h}\right) \in V_{h}^{2}
$$

It follows that

$$
\begin{aligned}
(\alpha-\Delta t)\left|\frac{u_{h}^{n+1}-u_{h}^{n}}{\Delta t}\right|^{2}+\frac{1}{2} a & \left(u_{h}^{n+1}, u_{h}^{n+1}\right)+\frac{1}{2} a\left(u_{h}^{n}, u_{h}^{n}\right) \\
& \leq R\left(u_{h}^{0}, u_{h}^{1}\right)+\beta a\left(u_{h}^{1}, u_{h}^{1}\right)+\beta a\left(u_{h}^{0}, u_{h}^{0}\right)+\sum_{p=1}^{n}\left|g^{p}\right|^{2} \Delta t+\sum_{p=0}^{n-1}\left|\frac{u_{h}^{p+1}-u_{h}^{p}}{\Delta t}\right|^{2} \Delta t .
\end{aligned}
$$

Since $\alpha-\Delta t \geq \alpha-\Delta t_{h}>0$, Grönwall's lemma implies that

$$
\sum_{p=0}^{n}\left|\frac{u_{h}^{p+1}-u_{h}^{p}}{\Delta t}\right|^{2} \leq\left|\frac{u_{h}^{1}-u_{h}^{0}}{\Delta t}\right|^{2} \exp \left(\frac{n \Delta t}{\alpha-\Delta t}\right)+\sum_{p=1}^{n} k_{p} \exp \left(\frac{(n-p) \Delta t}{\alpha-\Delta t}\right)
$$

with

$$
k_{p}=\frac{1}{\alpha-\Delta t}\left(R\left(u_{h}^{0}, u_{h}^{1}\right)+\beta a\left(u_{h}^{1}, u_{h}^{1}\right)+\beta a\left(u_{h}^{0}, u_{h}^{0}\right)+\sum_{k=1}^{p}\left|g^{k}\right|^{2} \Delta t\right) .
$$

Since $f \in L^{2}(0, T ; H)$, we infer that the right hand side of $(7)$ remains bounded by a constant which depends only on the data $\left(f, u_{0}, v_{0}\right)$.

We may observe that the lack of stability is due to the terms $(1-2 \beta) a\left(u_{h}^{n}, u_{h}^{n+1}\right)$ and $(1-2 \beta) a\left(u_{h}^{n-1}, u_{h}^{n}\right)$ which appear in the decomposition of $a\left(\beta u_{h}^{n+1}+(1-2 \beta) u_{h}^{n}+\beta u_{h}^{n-1}, u_{h}^{n-1}-u_{h}^{n+1}\right)$. For the case $\beta=1 / 2$, this difficulty does not occur and we obtain an unconditional stability result: 
Proposition 3.3. Let $\beta=1 / 2$. Then there exists a constant depending only on the data, $C\left(f, u_{0}, v_{0}\right)$, such that for all $h>0$ and for all $N \geq 1$

$$
\left|\frac{u_{h}^{n+1}-u_{h}^{n}}{\Delta t}\right|^{2}+\frac{1}{2} a\left(u_{h}^{n}, u_{h}^{n}\right)+\frac{1}{2} a\left(u_{h}^{n+1}, u_{h}^{n+1}\right) \leq C\left(f, u_{0}, v_{0}\right)
$$

for all $n \in\{1, \ldots, N-1\}$, where $\left(u_{h}^{n+1}\right)_{1 \leq n \leq N-1}$ are the solutions of problems $\left(P_{h \beta}^{n+1}\right)_{1 \leq n \leq N-1}$.

We define now an approximate solution $u_{h, N}^{\beta}(\beta \in[0,1 / 2])$ of problem $(P)$ by a linear interpolation of the solutions $u_{h}^{n+1}$ of $\left(P_{h \beta}^{n+1}\right)$. More precisely, for all $h>0$ and $N \geq 1$

$$
u_{h, N}^{\beta}(x, t)=u_{h}^{n} \frac{(n+1) \Delta t-t}{\Delta t}+u_{h}^{n+1} \frac{t-n \Delta t}{\Delta t},
$$

for all $t \in[n \Delta t,(n+1) \Delta t], 0 \leq n \leq N-1$.

Let $\alpha \in(0,1)$ and $N_{h}$ be defined by condition (6) if $\beta \in[0,1 / 2)$, otherwise let $N_{h}=1$ for all $h>0$. The previous stability results imply that there exists a subsequence, still denoted $\left(u_{h, N}^{\beta}\right)_{h>0, N \geq N_{h}}$, and $u \in W=$ $\left\{w \in L^{\infty}(0, T ; V), w_{t} \in L^{\infty}(0, T ; H)\right\}$ such that

$$
\begin{aligned}
& u_{h, N}^{\beta} \rightarrow u \quad \text { weakly* in } L^{\infty}(0, T ; V), \\
& \frac{\partial u_{h, N}^{\beta}}{\partial t} \rightarrow \frac{\partial u}{\partial t} \quad \text { weakly* in } L^{\infty}(0, T ; H) .
\end{aligned}
$$

With Simon's lemma [21] we infer that $W$ is compactly embedded in $C^{0}\left([0, T] ; H^{1}(0, L)\right)$ and we know also that $W \subset C^{0,1 / 2}([0, L] \times[0, T])[19]$. It follows that, possibly extracting another subsequence, we have

$$
u_{h, N}^{\beta} \rightarrow u \quad \text { strongly in } C^{0}\left([0, T] ; H^{1}(0, L)\right)
$$

and thus $u$ belongs to $L^{2}(0, T ; K)$ and $u(\cdot, 0)=u_{0}$.

Let us prove now that $u$ is a solution of problem $(P)$.

Theorem 3.4. Let $\beta \in[0,1 / 2]$ and let $N_{h}$ be defined by condition (6) if $\beta \neq 1 / 2$, otherwise $N_{h}=1$ for all $h>0$. The sequence of approximate solutions $\left(u_{h, N}^{\beta}\right)_{h>0, N \geq N_{h}}$ admits a subsequence which converges weakly* in $W$ to a solution of problem $(P)$.

Proof. We consider now the converging subsequence of $\left(u_{h, N}^{\beta}\right)_{h>0, N \geq N_{h}}$, still denoted $\left(u_{h, N}^{\beta}\right)_{h>0, N \geq N_{h}}$. Let $\tilde{w} \in \mathcal{H} \cap L^{2}(0, T ; K)$ such that $\tilde{w}(\cdot, T)=u(\cdot, T)$. We will prove that

$$
\begin{aligned}
& -\int_{0}^{T}\left(u_{t}(\cdot, t), \tilde{w}_{t}(\cdot, t)-u_{t}(\cdot, t)\right) \mathrm{d} t+\int_{0}^{T} a(u(\cdot, t), \tilde{w}(\cdot, t)-u(\cdot, t)) \mathrm{d} t \\
& \geq\left(v_{0}, \tilde{w}(\cdot, 0)-u_{0}\right)+\int_{0}^{T}(f(\cdot, t), \tilde{w}(\cdot, t)-u(\cdot, t)) \mathrm{d} t .
\end{aligned}
$$

In order to do so, we introduce a well-suited test-function $w_{h}=w_{h}^{n}$ in the problems $\left(P_{h \beta}^{n+1}\right)$ for $n=1$ to $N-1$ and we perform a discrete integration. Then we pass to the limit as $h$ and $\Delta t$ tend to zero. 
Step 1. Construction of a well-suited test-function $w_{h}^{n}$.

The most natural idea would be to define $w_{h}^{n}$ as the projection on $V_{h}$ of an approximate value of $w$ at time $t_{n}=n \Delta t(w$ is defined only for almost every $t)$ but unfortunately the projection does not preserve the unilateral constraints and this choice would not necessarily give a test-function in $K_{h}$ ! Thus we construct an auxiliary function $w^{\eta, \mu}$ as follows.

Let $\varepsilon \in(0, T / 2)$ and $\phi$ be a $C^{\infty}$-function such that

$$
\left\{\begin{array}{l}
0 \leq \phi(t) \leq 1 \quad \forall t \in[0, T], \\
\phi(t)=0 \quad \forall t \in[T-3 \varepsilon / 2, T], \quad \phi(t)=1 \quad \forall t \in[0, T-2 \varepsilon] .
\end{array}\right.
$$

We denote $w=(1-\phi) u+\phi \tilde{w}$. Since $K$ is convex, we have immediately $w \in \mathcal{H} \cap L^{2}(0, T ; K)$ and $w(\cdot, t)=u(\cdot, t)$ for all $t \in[T-3 \varepsilon / 2, T]$.

Let $\eta \in(0, \varepsilon / 2)$ and $\mu \in(0,1)$. Following the same ideas as in [19] we define $w^{\eta, \mu}$ by

$$
w^{\eta, \mu}(\cdot, t)=u(\cdot, t)+\frac{1}{\eta} \int_{t}^{t+\eta}((1-\mu) w(\cdot, s)-u(\cdot, s)) \mathrm{d} s \quad \forall t \in[0, T-\varepsilon / 2] .
$$

Since $u \in W$ and $w \in \mathcal{H}$, we have immediately $w^{\eta, \mu}-u \in C^{0}([0, T] ; V), w_{t}^{\eta, \mu} \in L^{2}(0, T ; H)$ and $w^{\eta, \mu} \in$ $L^{\infty}(0, T ; V) \cap C^{0}\left([0, T] ; H^{1}(0, L)\right)$. Moreover we can choose $\eta$ such that $w^{\eta, \mu}$ satisfies strictly the constraint. More precisely, for all $t \in[0, T-\varepsilon / 2]$ and for all $x \in[0, L]$ we have

$$
w^{\eta, \mu}(x, t)=\frac{1}{\eta} \int_{t}^{t+\eta}(1-\mu) w(x, s) \mathrm{d} s+u(x, t)-\frac{1}{\eta} \int_{t}^{t+\eta} u(x, s) \mathrm{d} s .
$$

The first term of the right hand side belongs to $\left[(1-\mu) g_{1}(x),(1-\mu) g_{2}(x)\right]$ with the convention that $(1-\mu) g_{i}(x)=$ $g_{i}(x)(i=1,2)$ if $g_{i}(x) \in\{+\infty,-\infty\}$, and recalling that $u \in C^{0,1 / 2}([0, L] \times[0, T])$ we have

$$
\left|u(x, t)-\frac{1}{\eta} \int_{t}^{t+\eta} u(x, s) \mathrm{d} s\right| \leq \frac{1}{\eta} \int_{t}^{t+\eta}|u(x, t)-u(x, s)| \mathrm{d} s \leq \frac{2 C_{0} \sqrt{\eta}}{3}
$$

where $C_{0}$ is the Hölder continuity coefficient of $u$.

Thus, remembering the constant $g>0$ in (3), we choose $\eta$ such that

$$
\frac{2 C_{0} \sqrt{\eta}}{3} \leq \frac{\mu}{2} g
$$

which ensures that

$$
g_{1}(x)+\frac{\mu}{2} g \leq w^{\eta, \mu}(x, t) \leq g_{2}(x)-\frac{\mu}{2} g
$$

for all $t \in[0, T-\varepsilon / 2]$ and for all $x \in[0, L]$, with the convention that $g_{i}(x) \pm \frac{\mu}{2} g=g_{i}(x)(i=1,2)$ if $g_{i}(x) \in\{+\infty,-\infty\}$.

Now, we assume that $\Delta t<\frac{\varepsilon}{2}$ and, for all $n \in\{1, \ldots, N-1\}$, we define the test-function $w_{h}^{n}$ by

$$
w_{h}^{n}=\left\{\begin{array}{l}
u_{h}^{n+1}+Q_{h}\left(w^{\eta, \mu}(\cdot, n \Delta t)-u(\cdot, n \Delta t)\right) \quad \text { if } n \Delta t \leq T-\varepsilon \\
u_{h}^{n+1} \quad \text { if } n \Delta t>T-\varepsilon
\end{array}\right.
$$

We have to check that $w_{h}^{n}$ belongs to $K_{h}$. 
Lemma 3.5. There exist $h_{1}>0$ and $N_{h}^{\prime} \geq N_{h}$ such that, for all $h \in\left(0, h_{1}\right)$ and for all $N \geq N_{h}^{\prime}$, we have

$$
w_{h}^{n} \in K_{h} \quad \forall n \in\{1, \ldots, N-1\} .
$$

Proof. First of all it is clear that $w_{h}^{n} \in V_{h}$ and $w_{h}^{n} \in K_{h}$ if $n \Delta t>T-\varepsilon$. Otherwise, when $n \Delta t \leq T-\varepsilon$, we rewrite $w_{h}^{n}$ as follows:

$$
\begin{aligned}
& w_{h}^{n}=u_{h, N}^{\beta}(\cdot,(n+1) \Delta t)-u(\cdot,(n+1) \Delta t)+u(\cdot,(n+1) \Delta t)-u(\cdot, n \Delta t) \\
& +w^{\eta, \mu}(\cdot, n \Delta t)+\left(Q_{h}-I\right)\left(w^{\eta, \mu}(\cdot, n \Delta t)-u(\cdot, n \Delta t)\right) .
\end{aligned}
$$

We already know that $\left(u_{h, N}^{\beta}\right)_{h>0, N \geq N_{h}}$ converges to $u$ strongly in $C^{0}\left([0, T] ; H^{1}(0, L)\right)$ and $u \in C^{0,1 / 2}([0, L] \times$ $[0, T])$, thus

$$
\sup _{x \in[0, L]}|u(x,(n+1) \Delta t)-u(x, n \Delta t)| \leq C_{0} \sqrt{\Delta t}
$$

and

$$
\sup _{x \in[0, L]}\left|u_{h, N}^{\beta}(x,(n+1) \Delta t)-u(x,(n+1) \Delta t)\right| \leq C_{1}\left\|u_{h, N}^{\beta}-u\right\|_{C^{0}\left([0, T] ; H^{1}(0, L)\right)}
$$

where $C_{1}$ is the norm of the canonical injection of $H^{1}(0, L)$ into $C^{0}([0, L])$. Moreover

$$
\begin{aligned}
& \sup _{x \in[0, L]}\left|\left(Q_{h}-I\right)\left(w^{\eta, \mu}(x, n \Delta t)-u(x, n \Delta t)\right)\right| \\
& \leq C_{1}\left\|\left(Q_{h}-I\right)\left(w^{\eta, \mu}(\cdot, n \Delta t)-u(\cdot, n \Delta t)\right)\right\|_{H^{1}(0, L)} \\
& \leq C_{1} \gamma_{h}\left\|w^{\eta, \mu}-u\right\|_{L^{\infty}(0, T ; V)} .
\end{aligned}
$$

By choosing $h_{1}$ and $N_{h}^{\prime} \geq N_{h}$ such that

$$
\frac{\mu}{2} g \geq C_{0} \sqrt{\Delta t}+C_{1}\left\|u_{h, N}^{\beta}-u\right\|_{C^{0}\left([0, T] ; H^{1}(0, L)\right)}+C_{1} \gamma_{h}\left\|w^{\eta, \mu}-u\right\|_{L^{\infty}(0, T ; V)}
$$

for all $h \in\left(0, h_{1}\right)$ and $\Delta t=\frac{T}{N}$ with $N \geq N_{h}^{\prime}$, relations (10) and (12)-(15) imply that $w_{h}^{n}(x)$ belongs to $\left[g_{1}(x), g_{2}(x)\right]$ for all $x \in[0, L]$ if $n \Delta t \leq T-\varepsilon$ which concludes the proof.

Remark 3.6. It should be noticed that it is essential that $w^{\eta, \mu}$ satisfies strictly the unilateral constraints since they are not preserved by the projection $Q_{h}$. From a "technical" point of view, the key point is the Hölder continuity of $u$ which allows us to choose $\eta$ such that relation (10) holds. This contruction of $w_{h}^{n}$ is inspired from [19] where the case of longitudinal vibrations is studied.

Step 2. Discrete integration and passage to the limit as $h$ and $\Delta t$ tend to zero.

Let us choose now $w_{h}=w_{h}^{n}$ in $\left(P_{h \beta}^{n+1}\right), 1 \leq n \leq N-1$ and define $N^{\prime}=\left\lfloor\frac{T-\varepsilon}{\Delta t}\right\rfloor$. With a discrete integration we obtain

$$
\begin{aligned}
& \left(\frac{u_{h}^{1}-u_{h}^{0}}{\Delta t}, w_{h}^{0}-u_{h}^{1}\right)+\sum_{n=1}^{N^{\prime}}\left(g^{n}, w_{h}^{n}-u_{h}^{n+1}\right) \Delta t \\
& \leq \sum_{n=1}^{N^{\prime}} a\left(\beta u_{h}^{n+1}+(1-2 \beta) u_{h}^{n}+\beta u_{h}^{n-1}, w_{h}^{n}-u_{h}^{n+1}\right) \Delta t \\
& -\sum_{n=1}^{N^{\prime}+1}\left(\frac{u_{h}^{n}-u_{h}^{n-1}}{\Delta t}, \frac{\left(w_{h}^{n}-u_{h}^{n+1}\right)-\left(w_{h}^{n-1}-u_{h}^{n}\right)}{\Delta t}\right) \Delta t
\end{aligned}
$$


and we have to pass to the limit in each term as $h$ and $\Delta t$ tend to zero. Recalling (5) we immediately infer that

$$
\left(\frac{u_{h}^{1}-u_{h}^{0}}{\Delta t}, w_{h}^{0}-u_{h}^{1}\right) \rightarrow\left(v_{0}, w^{\eta, \mu}(\cdot, 0)-u(\cdot, 0)\right)
$$

Then, we rewrite the second term as follows

$$
\begin{aligned}
\sum_{n=1}^{N^{\prime}}\left(g^{n}, w_{h}^{n}-u_{h}^{n+1}\right) \Delta t=\sum_{n=1}^{N^{\prime}}\left(f^{n},\right. & \left.w_{h}^{n}-u_{h}^{n+1}\right) \Delta t \\
& \quad+\beta \sum_{n=1}^{N^{\prime}+1}\left(f^{n}-f^{n-1},\left(w_{h}^{n-1}-u_{h}^{n}\right)-\left(w_{h}^{n}-u_{h}^{n+1}\right)\right) \Delta t+\beta\left(f^{0}-f^{1}, w_{h}^{0}-u_{h}^{1}\right) \Delta t .
\end{aligned}
$$

But

$$
\begin{aligned}
& \sum_{n=1}^{N^{\prime}}\left(f^{n}, w_{h}^{n}-u_{h}^{n+1}\right) \Delta t=\sum_{n=1}^{N^{\prime}} \int_{n \Delta t}^{(n+1) \Delta t}\left(f(\cdot, s),\left(w^{\eta, \mu}-u\right)(\cdot, s)\right) \mathrm{d} s \\
& +\sum_{n=1}^{N^{\prime}} \int_{n \Delta t}^{(n+1) \Delta t}\left(f(\cdot, s),\left(w^{\eta, \mu}-u\right)(\cdot, n \Delta t)-\left(w^{\eta, \mu}-u\right)(\cdot, s)\right) \mathrm{d} s \\
& +\sum_{n=1}^{N^{\prime}}\left(f^{n},\left(Q_{h}-I\right)\left(\left(w^{\eta, \mu}-u\right)(\cdot, n \Delta t)\right)\right) \Delta t .
\end{aligned}
$$

Observing that

$$
\left|\left(Q_{h}-I\right)\left(w^{\eta, \mu}-u\right)(\cdot, n \Delta t)\right| \leq \gamma_{h}\left\|w^{\eta, \mu}-u\right\|_{L^{\infty}(0, T ; V)}
$$

for all $n \in\left\{1, \ldots, N^{\prime}\right\}$, we obtain

$$
\begin{aligned}
\left|\sum_{n=1}^{N^{\prime}}\left(f^{n},\left(Q_{h}-I\right)\left(\left(w^{\eta, \mu}-u\right)(\cdot, n \Delta t)\right)\right) \Delta t\right| & \leq \sum_{n=1}^{N^{\prime}} \gamma_{h}\left|f^{n}\right|\left\|w^{\eta, \mu}-u\right\|_{L^{\infty}(0, T ; V)} \Delta t \\
& \leq \sqrt{T} \gamma_{h}\|f\|_{L^{2}(0, T ; H)}\left\|w^{\eta, \mu}-u\right\|_{L^{\infty}(0, T ; V)} \rightarrow 0 .
\end{aligned}
$$

Moreover, with the definition of $w^{\eta, \mu}$, we have

$$
\begin{aligned}
\|\left(w^{\eta, \mu}-u\right)(\cdot, n \Delta t) & \left.-\left(w^{\eta, \mu}-u\right)(\cdot, s)\right) \|_{V} \\
\leq \frac{1}{\eta} \int_{n \Delta t}^{s}\|((1-\mu) w-u)(\cdot, \sigma)\|_{V} d \sigma+\int_{n \Delta t+\eta}^{s+\eta}\|((1-\mu) w-u)(\cdot, \sigma)\|_{V} \mathrm{~d} \sigma & \leq \frac{2 \sqrt{s-n \Delta t}}{\eta}\|(1-\mu) w-u\|_{L^{2}(0, T ; V)}
\end{aligned}
$$

for all $n \in\left\{0, \cdots, N^{\prime}\right\}$ and $s \in[n \Delta t,(n+1) \Delta t]$. 
If we denote by $C$ the norm of the canonical injection of $\left(V,\|\cdot\|_{V}\right)$ into $(H,|\cdot|)$, we get

$$
\begin{aligned}
& \left|\sum_{n=1}^{N^{\prime}} \int_{n \Delta t}^{(n+1) \Delta t}\left(f(\cdot, s),\left(w^{\eta, \mu}-u\right)(\cdot, n \Delta t)-\left(w^{\eta, \mu}-u\right)(\cdot, s)\right) \mathrm{d} s\right| \\
& \leq \sqrt{\Delta t} \frac{2 C \sqrt{T}}{\eta}\|(1-\mu) w-u\|_{L^{2}(0, T ; V)}\|f\|_{L^{2}(0, T ; H)} \rightarrow 0 .
\end{aligned}
$$

Finally, since $f \in L^{2}(0, T ; H), w^{\eta, \mu}-u \in C^{0}([0, T] ; V) \subset L^{\infty}(0, T ; H)$ and $\left|T-\varepsilon-\left(N^{\prime}+1\right) \Delta t\right| \leq \Delta t$, we may conclude that

$$
\lim _{\Delta t \rightarrow 0} \sum_{n=1}^{N^{\prime}} \int_{n \Delta t}^{(n+1) \Delta t}\left(f(\cdot, s),\left(w^{\eta, \mu}-u\right)(\cdot, s)\right) \mathrm{d} s=\int_{0}^{T-\varepsilon}\left(f(\cdot, s),\left(w^{\eta, \mu}-u\right)(\cdot, s)\right) \mathrm{d} s
$$

and

$$
\sum_{n=1}^{N^{\prime}}\left(f^{n}, w_{h}^{n}-u_{h}^{n+1}\right) \Delta t \rightarrow \int_{0}^{T-\varepsilon}\left(f(\cdot, s),\left(w^{\eta, \mu}-u\right)(\cdot, s)\right) \mathrm{d} s
$$

Moreover, relation (17) implies that

$$
\left\|\left(w_{h}^{n-1}-u_{h}^{n}\right)-\left(w_{h}^{n}-u_{h}^{n+1}\right)\right\|_{V} \leq \frac{2 \sqrt{\Delta t}}{\eta}\|(1-\mu) w-u\|_{L^{2}(0, T ; V)}
$$

for all $n \in\left\{1, \ldots, N^{\prime}+1\right\}$. It follows that

$$
\left|\sum_{n=1}^{N^{\prime}+1}\left(f^{n}-f^{n-1},\left(w_{h}^{n-1}-u_{h}^{n}\right)-\left(w_{h}^{n}-u_{h}^{n+1}\right)\right) \Delta t\right| \leq \sqrt{\Delta t} \frac{4 C \sqrt{T}}{\eta}\|(1-\mu) w-u\|_{L^{2}(0, T ; V)}\|f\|_{L^{2}(0, T ; H)} .
$$

Then we observe that

$$
\left\|w_{h}^{n}-u_{h}^{n+1}\right\|_{V} \leq\left\|w^{\eta, \mu}-u\right\|_{L^{\infty}(0, T ; V)} \quad \forall n \in\left\{0, \ldots, N^{\prime}\right\}
$$

Hence

$$
\left|\left(f^{0}-f^{1}, w_{h}^{0}-u_{h}^{1}\right) \Delta t\right| \leq 2 C \sqrt{\Delta t}\left\|w^{\eta, \mu}-u\right\|_{L^{\infty}(0, T ; V)}\|f\|_{L^{2}(0, T ; H)}
$$

and we may conclude that

$$
\sum_{n=1}^{N^{\prime}}\left(g^{n}, w_{h}^{n}-u_{h}^{n+1}\right) \Delta t \rightarrow \int_{0}^{T-\varepsilon}\left(f(\cdot, s),\left(w^{\eta, \mu}-u\right)(\cdot, s)\right) \mathrm{d} s
$$


Let us study now the convergence of the first term of the right hand side of (16). First we rewrite it as follows:

$$
\begin{aligned}
& \sum_{n=1}^{N^{\prime}} a\left(\beta u_{h}^{n+1}+(1-2 \beta) u_{h}^{n}+\beta u_{h}^{n-1}, w_{h}^{n}-u_{h}^{n+1}\right) \Delta t \\
& =a\left(\frac{1-2 \beta}{2} u_{h}^{1}+\beta u_{h}^{0}, w_{h}^{0}-u_{h}^{1}\right) \Delta t \\
& +\sum_{n=1}^{N^{\prime}+1} a\left(\beta u_{h}^{n-1}+\frac{1-2 \beta}{2} u_{h}^{n},\left(w_{h}^{n}-u_{h}^{n+1}\right)-\left(w_{h}^{n-1}-u_{h}^{n}\right)\right) \Delta t \\
& +\sum_{n=1}^{N^{\prime}} a\left(\frac{u_{h}^{n+1}+u_{h}^{n}}{2}, w_{h}^{n}-u_{h}^{n+1}\right) \Delta t .
\end{aligned}
$$

With the Propositions 3.1 and 3.3 we know that $\left(\left\|u_{h, N}^{\beta}\right\|_{L^{\infty}(0, T ; V)}\right)_{h>0, N \geq N_{h}}$ is bounded independently of $h$ and $\Delta t$, thus

$$
\left|a\left(\frac{1-2 \beta}{2} u_{h}^{1}+\beta u_{h}^{0}, w_{h}^{0}-u_{h}^{1}\right)\right| \leq \frac{1}{2}\left\|u_{h, N}^{\beta}\right\|_{L^{\infty}(0, T ; V)}\left\|w^{\eta, \mu}-u\right\|_{L^{\infty}(0, T ; V)}
$$

and, with (18)

$$
\left|a\left(\beta u_{h}^{n-1}+\frac{1-2 \beta}{2} u_{h}^{n},\left(w_{h}^{n}-u_{h}^{n+1}\right)-\left(w_{h}^{n-1}-u_{h}^{n}\right)\right)\right| \leq \frac{\sqrt{\Delta t}}{\eta}\left\|u_{h, N}^{\beta}\right\|_{L^{\infty}(0, T ; V)}\|(1-\mu) w-u\|_{L^{2}(0, T ; V)} .
$$

Let us rewrite the last term of (19) as follows:

$$
\begin{aligned}
& \sum_{n=1}^{N^{\prime}} a\left(\frac{u_{h}^{n+1}+u_{h}^{n}}{2}, w_{h}^{n}-u_{h}^{n+1}\right) \Delta t=\sum_{n=1}^{N^{\prime}} \int_{n \Delta t}^{(n+1) \Delta t} a\left(u_{h, N}^{\beta}(\cdot, s), w_{h}^{n}-u_{h}^{n+1}\right) \mathrm{d} s \\
& \quad=-\int_{N^{\prime} \Delta t}^{T-\varepsilon} a\left(u_{h, N}^{\beta}(\cdot, s), Q_{h}\left(w^{\eta, \mu}-u\right)(\cdot, s)\right) \mathrm{d} s-\int_{0}^{\Delta t} a\left(u_{h, N}^{\beta}(\cdot, s), Q_{h}\left(w^{\eta, \mu}-u\right)(\cdot, s)\right) \mathrm{d} s \\
& \quad+\sum_{n=1}^{N^{\prime}} \int_{n \Delta t}^{(n+1) \Delta t} a\left(u_{h, N}^{\beta}(\cdot, s), Q_{h}\left(\left(w^{\eta, \mu}-u\right)(\cdot, n \Delta t)\right)-Q_{h}\left(\left(w^{\eta, \mu}-u\right)(\cdot, s)\right) \mathrm{d} s\right. \\
& \quad+\int_{0}^{T-\varepsilon} a\left(u_{h, N}^{\beta}(\cdot, s), Q_{h}\left(w^{\eta, \mu}-u\right)(\cdot, s)\right) \mathrm{d} s .
\end{aligned}
$$

The first two terms can be estimated by

$$
\left\|u_{h, N}^{\beta}\right\|_{L^{\infty}(0, T ; V)}\left\|w^{\eta, \mu}-u\right\|_{L^{\infty}(0, T ; V)} \Delta t
$$

and, with estimate (17) we have

$$
\begin{array}{r}
\left|\sum_{n=1}^{N^{\prime}} \int_{n \Delta t}^{(n+1) \Delta t} a\left(u_{h, N}^{\beta}(\cdot, s), Q_{h}\left(\left(w^{\eta, \mu}-u\right)(\cdot, n \Delta t)\right)-Q_{h}\left(\left(w^{\eta, \mu}-u\right)(\cdot, s)\right)\right) \mathrm{d} s\right| \\
\leq \frac{2 T \sqrt{\Delta t}}{\eta}\left\|u_{h, N}^{\beta}\right\|_{L^{\infty}(0, T ; V)}\|(1-\mu) w-u\|_{L^{2}(0, T ; V)} .
\end{array}
$$


Then, recalling that $Q_{h}\left(w^{\eta, \mu}-u\right)$ is the orthogonal projection of $w^{\eta, \mu}-u$ on $V_{h}$ with respect to the scalar product defined by $a$ on $V$, we obtain that

$$
\int_{0}^{T-\varepsilon} a\left(u_{h, N}^{\beta}(\cdot, s), Q_{h}\left(w^{\eta, \mu}-u\right)(\cdot, s)\right) \mathrm{d} s=\int_{0}^{T-\varepsilon} a\left(u_{h, N}^{\beta}(\cdot, s),\left(w^{\eta, \mu}-u\right)(\cdot, s)\right) \mathrm{d} s
$$

and the weak convergence of $u_{h}$ to $u$ in $L^{2}(0, T ; V)$ allows us to conclude that

$$
\sum_{n=1}^{N^{\prime}} a\left(\beta u_{h}^{n+1}+(1-2 \beta) u_{h}^{n}+\beta u_{h}^{n-1}, w_{h}^{n}-u_{h}^{n+1}\right) \Delta t \rightarrow \int_{0}^{T-\varepsilon} a\left(u(\cdot, s),\left(w^{\eta, \mu}-u\right)(\cdot, s)\right) \mathrm{d} s
$$

There remains now to study the convergence of the last term of (16) i.e.

$$
\sum_{n=1}^{N^{\prime}+1}\left(\frac{u_{h}^{n}-u_{h}^{n-1}}{\Delta t}, \frac{\left(w_{h}^{n}-u_{h}^{n+1}\right)-\left(w_{h}^{n-1}-u_{h}^{n}\right)}{\Delta t}\right) \Delta t
$$

In order to simplify the notations, let us define

$$
\psi_{\Delta t}(\cdot, t)=\frac{\left(w^{\eta, \mu}-u\right)(\cdot, t+\Delta t)-\left(w^{\eta, \mu}-u\right)(\cdot, t)}{\Delta t}, \quad \forall t \in[0, T-\varepsilon]
$$

We rewrite (20) as follows:

$$
\begin{aligned}
& \sum_{n=1}^{N^{\prime}+1}\left(\frac{u_{h}^{n}-u_{h}^{n-1}}{\Delta t}, \frac{\left(w_{h}^{n}-u_{h}^{n+1}\right)-\left(w_{h}^{n-1}-u_{h}^{n}\right)}{\Delta t}\right) \Delta t \\
& \quad=-\left(\frac{u^{N^{\prime}+1}-u^{N^{\prime}}}{\Delta t}, w_{h}^{N^{\prime}}-u_{h}^{N^{\prime}+1}\right) \\
& \quad+\sum_{n=1}^{N^{\prime}}\left(\frac{u_{h}^{n}-u_{h}^{n-1}}{\Delta t},\left(Q_{h}-I\right)\left(\psi_{\Delta t}(\cdot,(n-1) \Delta t)\right)\right) \Delta t \\
& \quad+\sum_{n=1}^{N^{\prime}} \int_{(n-1) \Delta t}^{n \Delta t}\left(\frac{u_{h}^{n}-u_{h}^{n-1}}{\Delta t}, \psi_{\Delta t}(\cdot,(n-1) \Delta t)-\psi_{\Delta t}(\cdot, t)\right) \mathrm{d} t \\
& \quad+\sum_{n=1}^{N^{\prime}} \int_{(n-1) \Delta t}^{n \Delta t}\left(\frac{u_{h}^{n}-u_{h}^{n-1}}{\Delta t}, \psi_{\Delta t}(\cdot, t)\right) \mathrm{d} t .
\end{aligned}
$$

The first term, which can be interpreted as a boundary term at $t=T$ for the discrete time integration, can be estimated by

$$
\left|\left(\frac{u^{N^{\prime}+1}-u^{N^{\prime}}}{\Delta t}, w_{h}^{N^{\prime}}-u_{h}^{N^{\prime}+1}\right)\right| \leq \frac{C}{\eta} \max _{1 \leq n \leq N}\left|\frac{u_{h}^{n}-u_{h}^{n-1}}{\Delta t}\right| \int_{N^{\prime} \Delta t}^{N^{\prime} \Delta t+\eta}\|(1-\mu) w(\cdot, s)-u(\cdot, s)\|_{V} \mathrm{~d} s .
$$

But $N^{\prime} \Delta t \geq T-3 \varepsilon / 2$, thus $w(\cdot, s)=u(\cdot, s)$ for all $s \in\left[N^{\prime} \Delta t, N^{\prime} \Delta t+\eta\right]$ and

$$
\int_{N^{\prime} \Delta t}^{N^{\prime} \Delta t+\eta}\|((1-\mu) w-u)(\cdot, s)\|_{V} \mathrm{~d} s=\int_{N^{\prime} \Delta t}^{N^{\prime} \Delta t+\eta} \mu\|u(\cdot, s)\|_{V} \mathrm{~d} s \leq \mu \eta\|u\|_{L^{\infty}(0, T ; V)} .
$$


Since Propositions 3.1 and 3.3 imply that $\max _{1 \leq n \leq N}\left|\frac{u_{h}^{n}-u_{h}^{n-1}}{\Delta t}\right|$ is bounded independently of $h$ and $\Delta t$, we infer that there exists a constant $C^{\prime}$ such that

$$
\left|\left(\frac{u_{h}^{N^{\prime}+1}-u_{h}^{N^{\prime}}}{\Delta t}, w_{h}^{N^{\prime}}-u_{h}^{N^{\prime}+1}\right)\right| \leq C^{\prime} \mu\|u\|_{L^{\infty}(0, T ; V)} .
$$

For the second term we perform the same kind of computation:

$$
\begin{aligned}
& \left|\sum_{n=1}^{N^{\prime}}\left(\frac{u_{h}^{n}-u_{h}^{n-1}}{\Delta t},\left(Q_{h}-I\right)\left(\psi_{\Delta t}(\cdot,(n-1) \Delta t)\right)\right) \Delta t\right| \\
& \leq \sum_{n=1}^{N^{\prime}} \gamma_{h}\left|\frac{u_{h}^{n}-u_{h}^{n-1}}{\Delta t}\right|\left\|\left(w^{\eta, \mu}-u\right)(\cdot, n \Delta t)-\left(w^{\eta, \mu}-u\right)(\cdot,(n-1) \Delta t)\right\|_{V} \\
& \leq \leq \frac{\gamma_{h}}{\eta} \max _{1 \leq n \leq N}\left|\frac{u_{h}^{n}-u_{h}^{n-1}}{\Delta t}\right| \sum_{n=1}^{N^{\prime}}\left(\int_{(n-1) \Delta t}^{n \Delta t}\|((1-\mu) w-u)(\cdot, s)\|_{V} \mathrm{~d} s\right. \\
& \left.\quad+\int_{(n-1) \Delta t+\eta}^{n \Delta t+\eta}\|((1-\mu) w-u)(\cdot, s)\|_{V} \mathrm{~d} s\right) \\
& \leq 2 \frac{\gamma_{h} \sqrt{T}}{\eta} \max _{1 \leq n \leq N}\left|\frac{u_{h}^{n}-u_{h}^{n-1}}{\Delta t}\right|\|(1-\mu) w-u\|_{L^{2}(0, T ; V)} .
\end{aligned}
$$

Recalling that $\left(\gamma_{h}\right)_{h>0}$ converges to zero, we obtain

$$
\sum_{n=1}^{N^{\prime}}\left(\frac{u_{h}^{n}-u_{h}^{n-1}}{\Delta t},\left(Q_{h}-I\right)\left(\psi_{\Delta t}(\cdot,(n-1) \Delta t)\right)\right) \Delta t \rightarrow 0 .
$$

In order to estimate the third term, we transform $\psi_{\Delta t}(\cdot,(n-1) \Delta t)-\psi_{\Delta t}(\cdot, t)$ as follows:

$$
\begin{aligned}
\psi_{\Delta t}(\cdot,(n-1) \Delta t)-\psi_{\Delta t}(\cdot, t) & \\
= & \frac{1}{\eta \Delta t}\left(\int_{(n-1) \Delta t}^{t}(((1-\mu) w-u)(\cdot, s+\Delta t)-((1-\mu) w-u)(\cdot, s)) \mathrm{d} s\right. \\
& \left.+\int_{t+\eta}^{(n-1) \Delta t+\eta}(((1-\mu) w-u)(\cdot, s+\Delta t)-((1-\mu) w-u)(\cdot, s)) \mathrm{d} s\right) \\
= & \frac{1}{\eta \Delta t} \int_{(n-1) \Delta t}^{t} \int_{s}^{s+\Delta t}\left((1-\mu) w_{t}-u_{t}\right)(\cdot, \sigma) \mathrm{d} \sigma \mathrm{d} s \\
& +\frac{1}{\eta \Delta t} \int_{t+\eta}^{(n-1) \Delta t+\eta} \int_{s}^{s+\Delta t}\left((1-\mu) w_{t}-u_{t}\right)(\cdot, \sigma) \mathrm{d} \sigma \mathrm{d} s .
\end{aligned}
$$

Hence

$$
\left|\psi_{\Delta t}(\cdot,(n-1) \Delta t)-\psi_{\Delta t}(\cdot, t)\right| \leq \frac{2(t-(n-1) \Delta t)}{\eta \sqrt{\Delta t}}\left\|(1-\mu) w_{t}-u_{t}\right\|_{L^{2}(0, T ; H)}
$$


and

$$
\begin{aligned}
& \left|\sum_{n=1}^{N^{\prime}} \int_{(n-1) \Delta t}^{n \Delta t}\left(\frac{u_{h}^{n}-u_{h}^{n-1}}{\Delta t}, \psi_{\Delta t}(\cdot,(n-1) \Delta t)-\psi_{\Delta t}(\cdot, t)\right) \mathrm{d} t\right| \\
& \quad \leq \sum_{n=1}^{N^{\prime}} \frac{\Delta t^{2}}{\eta \sqrt{\Delta t}}\left|\frac{u_{h}^{n}-u_{h}^{n-1}}{\Delta t}\right|\left\|(1-\mu) w_{t}-u_{t}\right\|_{L^{2}(0, T ; H)} \\
& \quad \leq \frac{T \sqrt{\Delta t}}{\eta} \max _{1 \leq n \leq N}\left|\frac{u_{h}^{n}-u_{h}^{n-1}}{\Delta t}\right|\left\|(1-\mu) w_{t}-u_{t}\right\|_{L^{2}(0, T ; H)} \rightarrow 0 .
\end{aligned}
$$

Finally, we observe that

$$
\begin{aligned}
& \sum_{n=1}^{N^{\prime}} \int_{(n-1) \Delta t}^{n \Delta t}\left(\frac{u_{h}^{n}-u_{h}^{n-1}}{\Delta t}, \psi_{\Delta t}(\cdot, t)\right) \mathrm{d} t=\int_{0}^{T-\varepsilon}\left(\frac{\partial u_{h, N}^{\beta}}{\partial t}(\cdot, t), \psi_{\Delta t}(\cdot, t)\right) \mathrm{d} t \\
& -\int_{N^{\prime} \Delta t}^{T-\varepsilon}\left(\frac{u_{h}^{N^{\prime}}-u_{h}^{N^{\prime}-1}}{\Delta t}, \psi_{\Delta t}(\cdot, t)\right) \mathrm{d} t .
\end{aligned}
$$

Since $(1-\mu) w-u \in L^{2}(0, T ; V)$, and

$$
\psi_{\Delta t}(\cdot, t)=\frac{1}{\eta \Delta t}\left(\int_{t+\eta}^{t+\eta+\Delta t}((1-\mu) w-u)(\cdot, s) \mathrm{d} s-\int_{t}^{t+\Delta t}((1-\mu) w-u)(\cdot, s) \mathrm{d} s\right)
$$

we obtain that

$$
\psi_{\Delta t}(\cdot, t) \rightarrow_{\Delta t \rightarrow 0} \frac{((1-\mu) w-u)(\cdot, t+\eta)-((1-\mu) w-u)(\cdot, t)}{\eta}
$$

strongly in $L^{2}(0, T-\varepsilon ; V)$. Since $\frac{\partial u_{h, N}^{\beta}}{\partial t}$ converges weakly to $\frac{\partial u}{\partial t}$ in $L^{2}(0, T ; V)$, it follows that

$$
\int_{0}^{T-\varepsilon}\left(\frac{\partial u_{h, N}^{\beta}}{\partial t}(\cdot, t), \psi_{\Delta t}(\cdot, t)\right) \mathrm{d} t \rightarrow \int_{0}^{T-\varepsilon}\left(\frac{\partial u}{\partial t}(\cdot, t), \frac{((1-\mu) w-u)(\cdot, t+\eta)-((1-\mu) w-u)(\cdot, t)}{\eta}\right) \mathrm{d} t .
$$

Moreover, for all $t \in[0, T-\varepsilon]$

$$
\left|\psi_{\Delta t}(\cdot, t)\right| \leq \frac{C}{\Delta t}\left\|\left(w^{\eta, \mu}-u\right)(\cdot, t+\Delta t)-\left(w^{\eta, \mu}-u\right)(\cdot, t)\right\|_{V} \leq \frac{2 C}{\eta \sqrt{\Delta t}}\|(1-\mu) w-u\|_{L^{2}(0, T ; V)}
$$

Thus

$$
\left|\int_{N^{\prime} \Delta t}^{T-\varepsilon}\left(\frac{u_{h}^{N^{\prime}}-u_{h}^{N^{\prime}-1}}{\Delta t}, \psi_{\Delta t}(\cdot, t)\right) \mathrm{d} t\right| \leq \frac{2 C \sqrt{\Delta t}}{\eta} \max _{1 \leq n \leq N}\left|\frac{u_{h}^{n}-u_{h}^{n-1}}{\Delta t}\right|\|(1-\mu) w-u\|_{L^{2}(0, T ; V)} .
$$


Taking into account the previous convergence results, we obtain

$$
\begin{aligned}
\int_{0}^{T-\varepsilon}\left(f(\cdot, t),\left(w^{\eta, \mu}-u\right)(\cdot, t)\right) \mathrm{d} t+\left(v_{0},\left(w^{\eta, \mu}-u\right)(\cdot, 0)\right) \leq \int_{0}^{T-\varepsilon} a\left(u(\cdot, t),\left(w^{\eta, \mu}-u\right)(\cdot, t)\right) \mathrm{d} t \\
-\int_{0}^{T-\varepsilon}\left(\frac{\partial u}{\partial t}(\cdot, t), \frac{((1-\mu) w-u)(\cdot, t+\eta)-((1-\mu) w-u)(\cdot, t)}{\eta}\right) \mathrm{d} t+C^{\prime} \mu\|u\|_{L^{\infty}(0, T ; V)}
\end{aligned}
$$

for all $\varepsilon \in(0, T / 2), \mu \in(0,1)$ and $\eta \in(0, \varepsilon / 2)$ satisfying (9).

Rewriting $((1-\mu) w-u)(\cdot, t+\eta)-((1-\mu) w-u)(\cdot, t)$ as

$$
\int_{t}^{t+\eta}\left((1-\mu) w_{t}-u_{t}\right)(\cdot, \sigma) \mathrm{d} \sigma
$$

and recalling that $(1-\mu) w_{t}-u_{t} \in L^{2}(0, T ; H)$, we obtain that

$$
\frac{1}{\eta} \int_{t}^{t+\eta}\left((1-\mu) w_{t}-u_{t}\right)(\sigma, \cdot) \mathrm{d} \sigma \rightarrow_{\eta \rightarrow 0}(1-\mu) w_{t}-u_{t}
$$

strongly in $L^{2}(0, T-\varepsilon ; H)$. Similarly, since $(1-\mu) w-u \in L^{2}(0, T ; V) \cap C^{0}([0, T] ; H)$, we have

$$
\left(w^{\eta, \mu}-u\right)(\cdot, t)=\frac{1}{\eta} \int_{t}^{t+\eta}((1-\mu) w-u)(\cdot, s) \mathrm{d} s \rightarrow_{\eta \rightarrow 0}((1-\mu) w-u)
$$

strongly in $L^{2}(0, T-\varepsilon ; V)$, and

$$
\left(w^{\eta, \mu}-u\right)(\cdot, 0) \rightarrow_{\eta \rightarrow 0}(1-\mu) w(\cdot, 0)-u(\cdot, 0) \quad \text { strongly in } H .
$$

Thus, when $\eta$ tends to zero, we get

$$
\begin{aligned}
& \int_{0}^{T-\varepsilon}(f(\cdot, t),((1-\mu) w-u)(\cdot, t)) \mathrm{d} t+\left(v_{0},((1-\mu) w-u)(\cdot, 0)\right) \\
& \leq \int_{0}^{T-\varepsilon} a(u(\cdot, t),((1-\mu) w-u)(\cdot, t)) \mathrm{d} t \\
& -\int_{0}^{T-\varepsilon}\left(\frac{\partial u}{\partial t}(\cdot, t),\left((1-\mu) w_{t}-u_{t}\right)(\cdot, t)\right) \mathrm{d} t+C^{\prime} \mu\|u\|_{L^{\infty}(0, T ; V)}
\end{aligned}
$$

Then we can pass to the limit when $\mu$ and $\varepsilon$ tend to zero and, observing that $w-u=\phi(\tilde{w}-u)$, we may conclude the proof.

\section{Finite element implementation of $\left(P_{h \beta}^{n+1}\right)$}

We present now some simulations when the contact with the obstacles takes place only at the right end of the beam, i.e.

$$
\left\{\begin{array}{l}
g_{1}(x)=-\infty, \quad g_{2}(x)=+\infty \quad \forall x \in[0, L) \\
g_{1}(L)=-g, \quad g_{2}(L)=g
\end{array}\right.
$$

with $g$ a positive real number. We use the P3 finite element approximation described at Section 2 . Let us recall that interval $[0, L]$ is decomposed into $J$ subintervals of length $h$ and that we denote by $\left(\varphi_{i}\right)_{1 \leq i \leq 2 J}$ the Hermite basis functions. 
Let $N \geq 1, \beta \in[0,1 / 2]$. For all $n \in\{0, \ldots, N-1\}$, the discretized problem $\left(P_{h \beta}^{n+1}\right)$ is given by:

$$
\left\{\begin{array}{l}
\text { find } \bar{u}^{n+1} \in \bar{K}_{h}=\mathbb{R}^{2 J-2} \times[-g, g] \times \mathbb{R} \text { such that, for all } \bar{w} \in \bar{K}_{h} \\
\left(\mathcal{M}\left(\frac{\bar{u}^{n+1}-2 \bar{u}^{n}+\bar{u}^{n-1}}{\Delta t^{2}}\right), \bar{w}-\bar{u}^{n+1}\right) \\
+\left(\mathcal{S}\left(\beta \bar{u}^{n+1}+(1-2 \beta) \bar{u}^{n}+\beta \bar{u}^{n-1}\right), \bar{w}-\bar{u}^{n+1}\right) \geq\left(G^{n}, \bar{w}-\bar{u}^{n+1}\right)
\end{array}\right.
$$

where

$$
G_{i}^{n}=\left(\beta f^{n+1}+(1-2 \beta) f^{n}+\beta f^{n-1}, \varphi_{i}\right) \quad i=1, \ldots, 2 J
$$

and $\mathcal{M}$ and $\mathcal{S}$ are respectively the global mass and stiffness matrices. The previous inequality is also equivalent to the differential inclusion:

$$
\mathcal{M}\left(\frac{\bar{u}^{n+1}-2 \bar{u}^{n}+\bar{u}^{n-1}}{\Delta t^{2}}\right)+\mathcal{S}\left(\beta \bar{u}^{n+1}+(1-2 \beta) \bar{u}^{n}+\beta \bar{u}^{n-1}\right)+\partial \psi_{\bar{K}_{h}}\left(\bar{u}^{n+1}\right) \ni G^{n}
$$

which can be rewritten as

$$
\left(\mathcal{M}+\Delta t^{2} \beta \mathcal{S}\right) \bar{u}^{n+1}+\Delta t^{2} \partial \psi_{\bar{K}_{h}}\left(\bar{u}^{n+1}\right) \ni F^{n}
$$

with $F^{n}=\left(2 \mathcal{M}-\Delta t^{2}(1-2 \beta) \mathcal{S}\right) \bar{u}^{n}-\left(\mathcal{M}+\Delta t^{2} \beta \mathcal{S}\right) \bar{u}^{n-1}+\Delta t^{2} G^{n}$.

More precisely, if we consider a single beam element $\left[x_{i}, x_{i+1}\right]$ of length $h(i=0, \ldots, J-1)$, the elemental mass and stiffness matrices are

$$
\mathcal{M}_{e}=\frac{h}{420}\left(\begin{array}{cccc}
156 & 22 h & 54 & -13 h \\
22 h & 4 h^{2} & 13 h & -3 h^{2} \\
54 & 13 h & 156 & -22 h \\
-13 h & -3 h^{2} & -22 h & 4 h^{2}
\end{array}\right), \mathcal{S}_{e}=\frac{2 k^{2}}{h^{3}}\left(\begin{array}{cccc}
6 & 3 h & -6 & 3 h \\
3 h & 2 h^{2} & -3 h & h^{2} \\
-6 & -3 h & 6 & -3 h \\
3 h & h^{2} & -3 h & 2 h^{2}
\end{array}\right)
$$

and the global system (21) is obtained as an assembly of the previous elemental matrices.

Recalling that $\partial \psi_{\bar{K}_{h}}\left(\bar{u}^{n+1}\right)$ is defined by

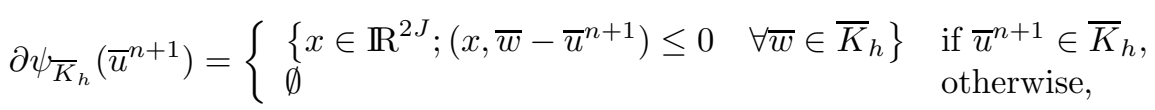

we infer immediately that $\bar{u}^{n+1}$ is the projection of $\left(\mathcal{M}+\Delta t^{2} \beta \mathcal{S}\right)^{-1} F^{n}$ on $\bar{K}_{h}$ with respect to the scalar metric defined by the matrix $\mathcal{A}=\left(\mathcal{M}+\Delta t^{2} \beta \mathcal{S}\right)$. From a numerical point of view, this projection can be quite easily computed. Indeed, we can apply the following lemma with $\mathcal{A}=\left(\mathcal{M}+\Delta t^{2} \beta \mathcal{S}\right), \lambda=\Delta t^{2}$ and $f=F^{n}$.

Lemma 4.1. [12], [3] Let $\mathcal{A}$ be a symmetric positive definite $2 J \times 2 J$ real matrix, $f \in \mathbb{R}^{2 J}$ and $u^{\prime}$ be the solution of $\mathcal{A} u^{\prime}=f$. Then, for all $\lambda>0$, the system

$$
\mathcal{A} \bar{u}+\lambda \partial \psi_{\bar{K}_{h}}(\bar{u}) \ni f
$$

with $\bar{K}_{h}=\mathbb{R}^{2 J-2} \times[-g, g] \times \mathbb{R}$ admits an unique solution $\bar{u}$ given by

$$
\bar{u}_{2 J-1}=\operatorname{Proj}\left(u_{2 J-1}^{\prime},[-g, g]\right),
$$

and $\left(\bar{u}_{k}\right)_{k \in\{1, \ldots, 2 J\} \backslash\{2 J-1\}}$ is solution of the truncated linear system

$$
(\mathcal{A} \bar{u})_{i}=f_{i}, \quad \text { for } i \in\{1, \ldots, 2 J\} \backslash\{2 J-1\} .
$$



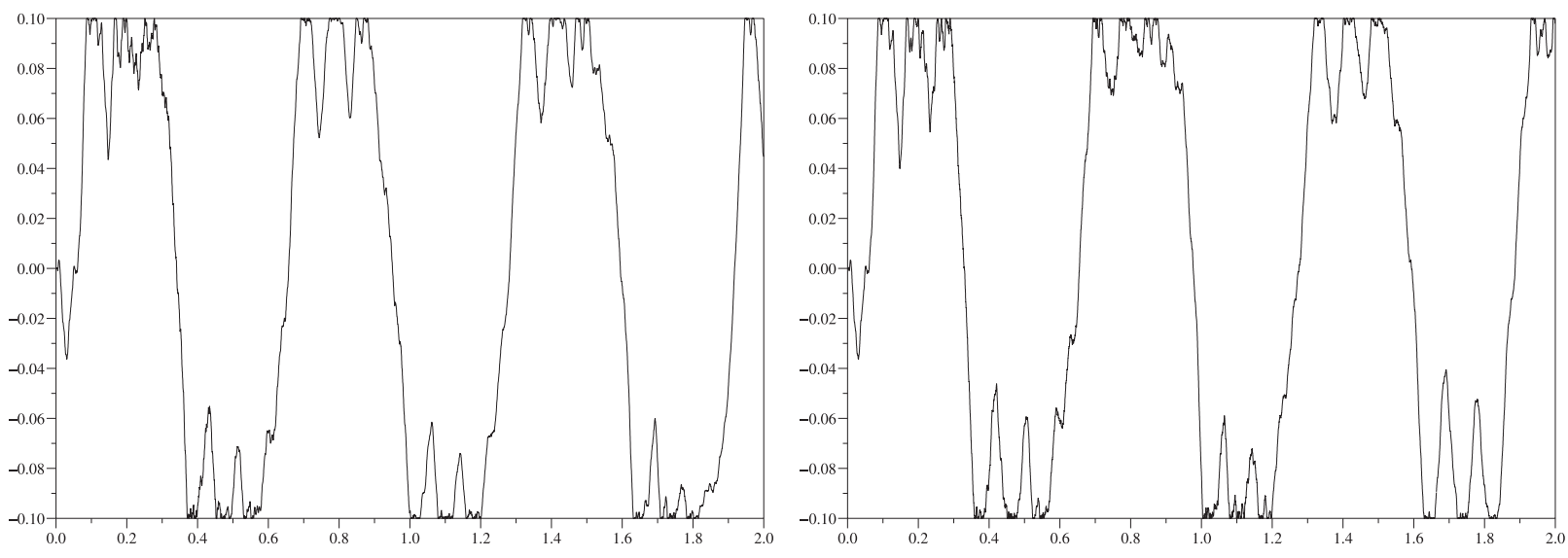

Figure $3 . \beta=\frac{1}{2}, \Delta t=5 \times 10^{-5} \mathrm{~s}$, and $\Delta t=5 \times 10^{-6} \mathrm{~s}$.
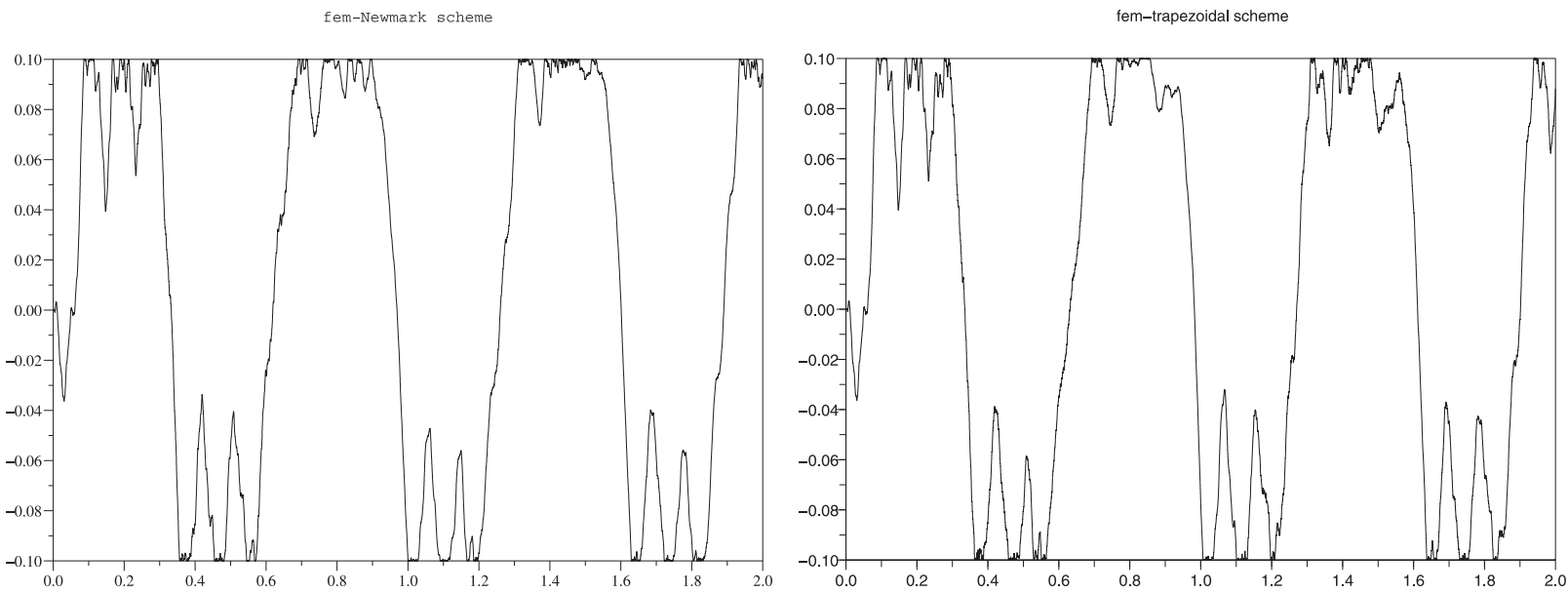

Figure 4. $\Delta t=10^{-6} \mathrm{~s}, \beta=\frac{1}{2}$ and $\beta=\frac{1}{4}$.

Consequently, at each time step, we compute the solution $\bar{u}^{n+1}$ of $\left(P_{h \beta}^{n+1}\right)$ by solving at most two linear systems. We may observe that the projection procedure $(22)$ ensures that $\bar{u}^{n+1} \in \bar{K}_{h}$.

We consider a steel pipe of length $L=1.501 \mathrm{~m}$, with an external diameter equal to $1 \mathrm{~cm}$ and a thickness equal to $0.5 \mathrm{~mm}$. Thus $k^{2}=\frac{E I}{\rho S}=282.84 \mathrm{~m}^{4} \cdot \mathrm{s}^{-2}$ where $E=2 \times 10^{11} \mathrm{~Pa}$ is the Young's modulus, $\rho=8 \times 10^{3} \mathrm{~kg} \cdot \mathrm{m}^{-3}$ is the material density, $S$ is the cross-section and $I$ the cross-sectional moment of inertia of the pipe (see also [12] for a more detailed description of the mechanical setting). The vibration of the support is given by $\phi(t)=0.2 \sin (10 t)$ for all $t \geq 0, g=0.1$ and the initial data are $u_{0}=0, v_{0}=-2 h$, i.e. $\tilde{u}(\cdot, 0)=\tilde{u}_{t}(\cdot, 0)=0($ at $t=0$ the beam is at rest).

In the next figures we show the approximate motion of the impacting end of the beam. The results given at Figures 3 and $4 \mathrm{a}$ have been obtained with $\beta=1 / 2, J=19$ and $\Delta t=5 \times 10^{-5} \mathrm{~s}, \Delta t=5 \times 10^{-6} \mathrm{~s}$ and $\Delta t=10^{-6} \mathrm{~s}$ (let us recall that we have unconditional stability for this value of $\beta$ ). Then, we present different results obtained with $J=19$ and $\beta=1 / 4$, which correspond to the well-known trapezoidal scheme, that is 

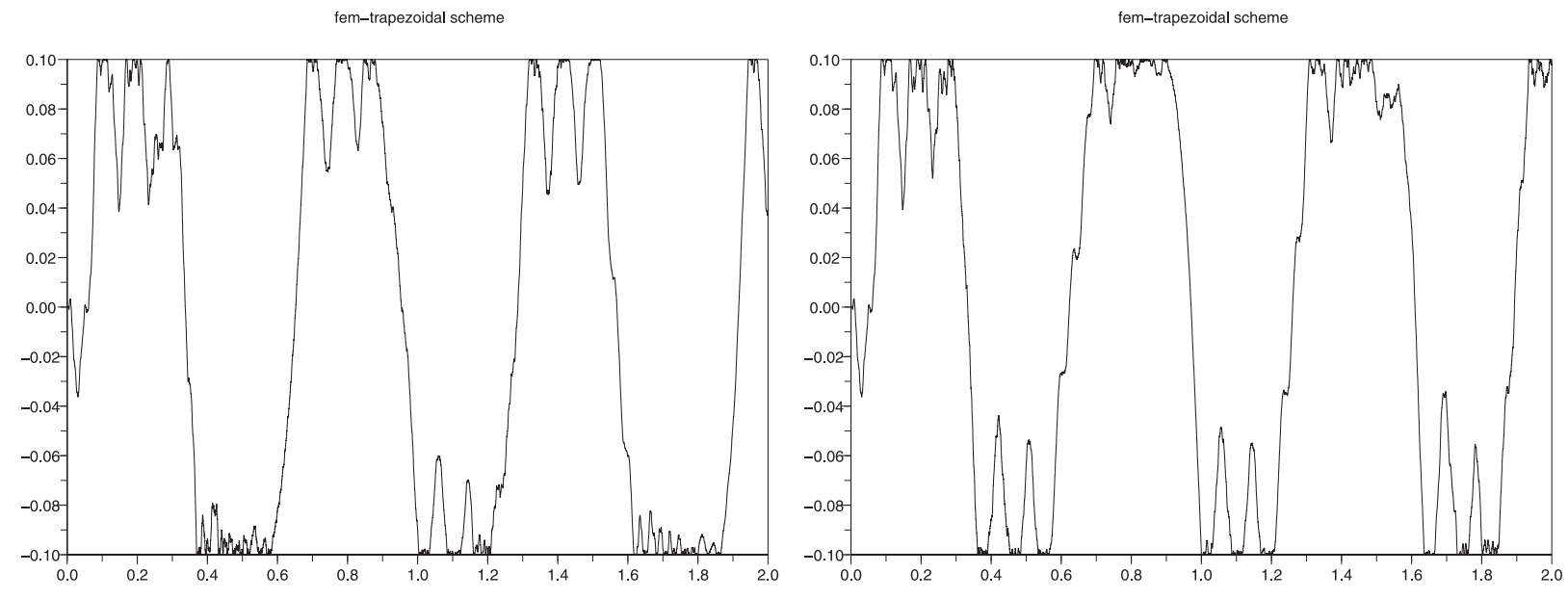

Figure $5 . \beta=\frac{1}{4}, \Delta t=5 \times 10^{-7} \mathrm{~s}$ and $\Delta t=2 \times 10^{-7} \mathrm{~s}$.

energy conservative when the constraints are not active. In that case, the stability condition leads to

$$
\Delta t<\sqrt{\frac{4}{\tilde{\kappa}(h)}}=2.733 \times 10^{-6} \mathrm{~s}
$$

with $\tilde{\kappa}(h)$ given by (25) (see the Appendix), and thus we will consider the following time-steps: $\Delta t=10^{-6} \mathrm{~s}$, $\Delta t=5 \times 10^{-7} \mathrm{~s}$ and $\Delta t=2 \times 10^{-7} \mathrm{~s}$ (see Fig. $4 \mathrm{~b}$ and 5 ).

We can observe that the trajectories are almost the same at the beginning of the time interval (up to the end of the first "contact period" i.e. $0 \leq t \leq 0.2)$ and remain quite similar afterwards even if the details of the impact phenomenon are different. This is not surprising since vibrations with unilateral constraints always lead to sensitivity to initial data. In this context we believe that the order of convergence is not a relevant information.

The motion of the impacting end of the beam has also been computed by using the normal compliance approximation of Signorini's conditions. In this case we have to define the penalty parameter $\varepsilon$. Although the corresponding stiffness $1 / \varepsilon$ has a physical meaning, the range of values usually chosen is quite large: $1 / \varepsilon=$ $10^{10} \mathrm{~N} \cdot \mathrm{m}^{-1}$ in [17], $1 / \varepsilon=5.5 \times 10^{7} \mathrm{~N} \cdot \mathrm{m}^{-1}$ in [22] for instance. In the following results we consider $1 / \varepsilon=$ $10^{8} \mathrm{~N} . \mathrm{m}^{-1}$ and we apply once again a Newmark's scheme with $J=19$ and $\Delta t=5 \times 10^{-6} \mathrm{~s}, \Delta t=10^{-6} \mathrm{~s}$, $\Delta t=5 \times 10^{-7} \mathrm{~s}$ and $\Delta t=2 \times 10^{-7} \mathrm{~s}$ (see Figs. 6, 7a, 8a and 9a). We should notice that we have to solve now a partial differential equation, thus we first choose $\beta=1 / 4$ for which the unconditional stability of Newmark's scheme and conservation of energy hold.

Let us recall that, despite the solutions of the "continuous" penalized problems $\left(\mathrm{P}_{\varepsilon}\right)$ converge to a solution of $(\mathrm{P})$, some numerical instabilities could happen when we compute approximate solutions of $\left(\mathrm{P}_{\varepsilon}\right)$. Such difficulties have already been observed in the case of a simplified model of vibrations (see [15]) and seem to be caused by a sensitivity of the approximate motion with respect to $\varepsilon$.

Here, for $\beta=\frac{1}{4}$, we observe that spurious high frequencies appear during "contact periods" (Fig. 6) and this phenomenon can be controlled only for very small time steps. For $\beta=\frac{1}{2}$, the results are worse: for $\Delta t=5 \times 10^{-6}$, the solution becomes quickly instable (after the first impact period) and, even if the time-step decays, some instabilities still remain (see for instance Figs. 7b, 8b and 9b). 


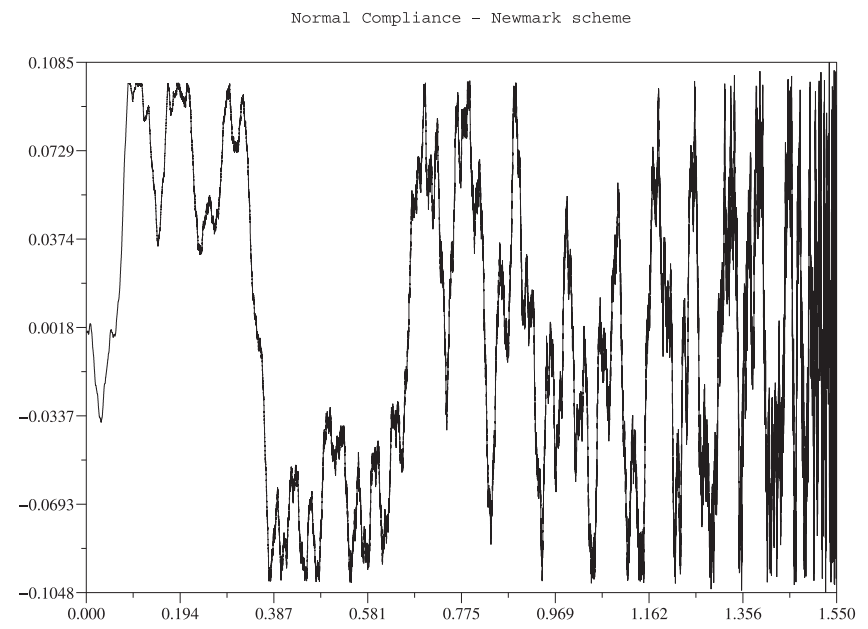

Figure $6 . \Delta t=5 \times 10^{-6} \mathrm{~s}$ and $\beta=\frac{1}{4}$.
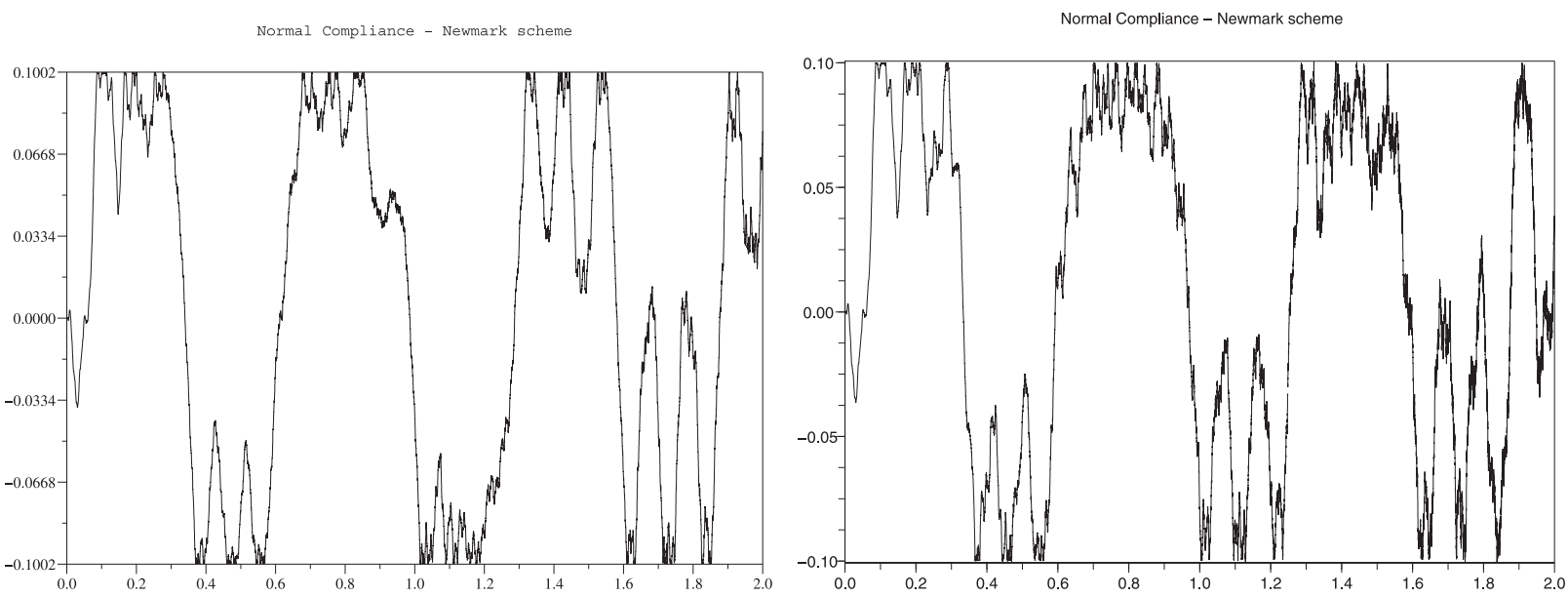

Figure 7. $\Delta t=10^{-6} \mathrm{~s}, \beta=\frac{1}{4}$ and $\beta=\frac{1}{2}$.

Moreover, the non-penetration condition is violated by the trajectories computed with the normal compliance approximation while it remains satisfied by the approximate motions $u_{h, N}^{\beta}$. More precisely we have

$$
\begin{aligned}
& \max _{0 \leq t \leq 2}\left|u_{a p p}(L, t)-g\right|=1.968 \times 10^{-4} \mathrm{~m} \quad \text { for } \Delta t=2 \times 10^{-7} \mathrm{~s} \text { and } \beta=\frac{1}{4} \\
& \max _{0 \leq t \leq 2}\left|u_{a p p}(L, t)-g\right|=2.143 \times 10^{-4} \mathrm{~m} \quad \text { for } \Delta t=2 \times 10^{-7} \mathrm{~s} \text { and } \beta=\frac{1}{2}
\end{aligned}
$$



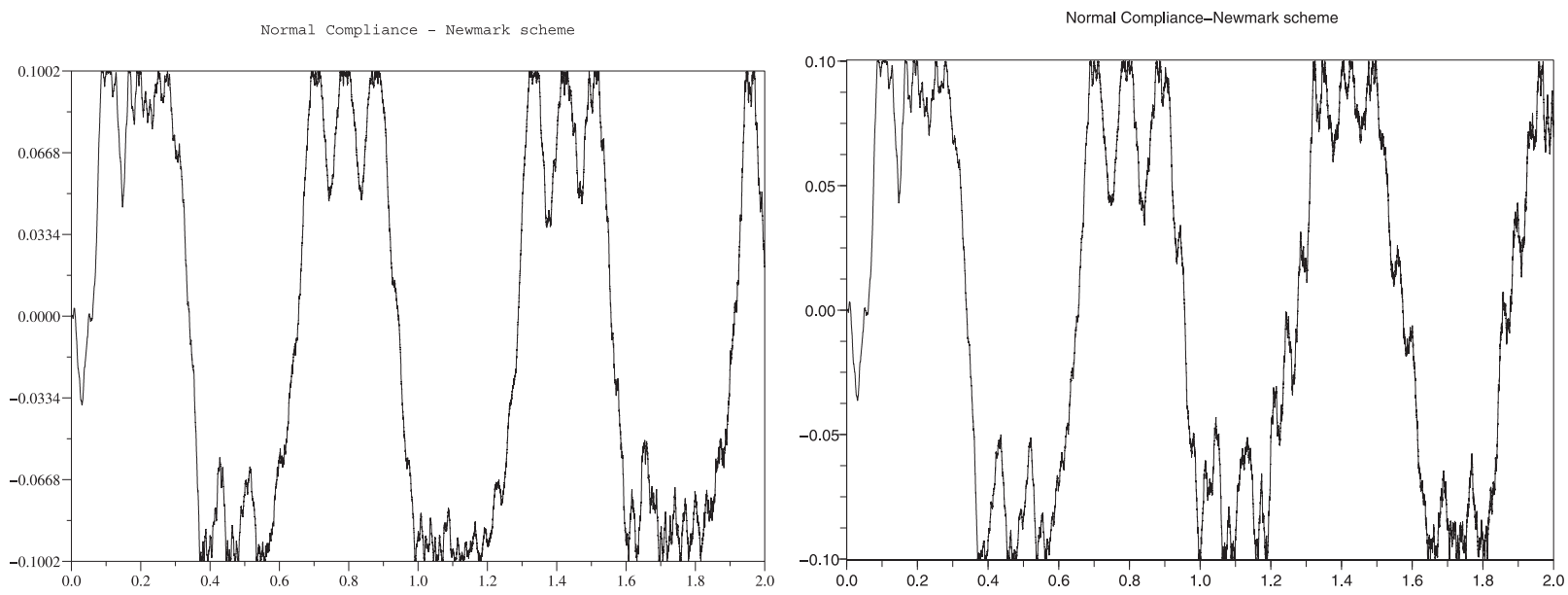

FiguRE 8. $\Delta t=5 \times 10^{-7} \mathrm{~s}, \beta=\frac{1}{4}$ and $\beta=\frac{1}{2}$.
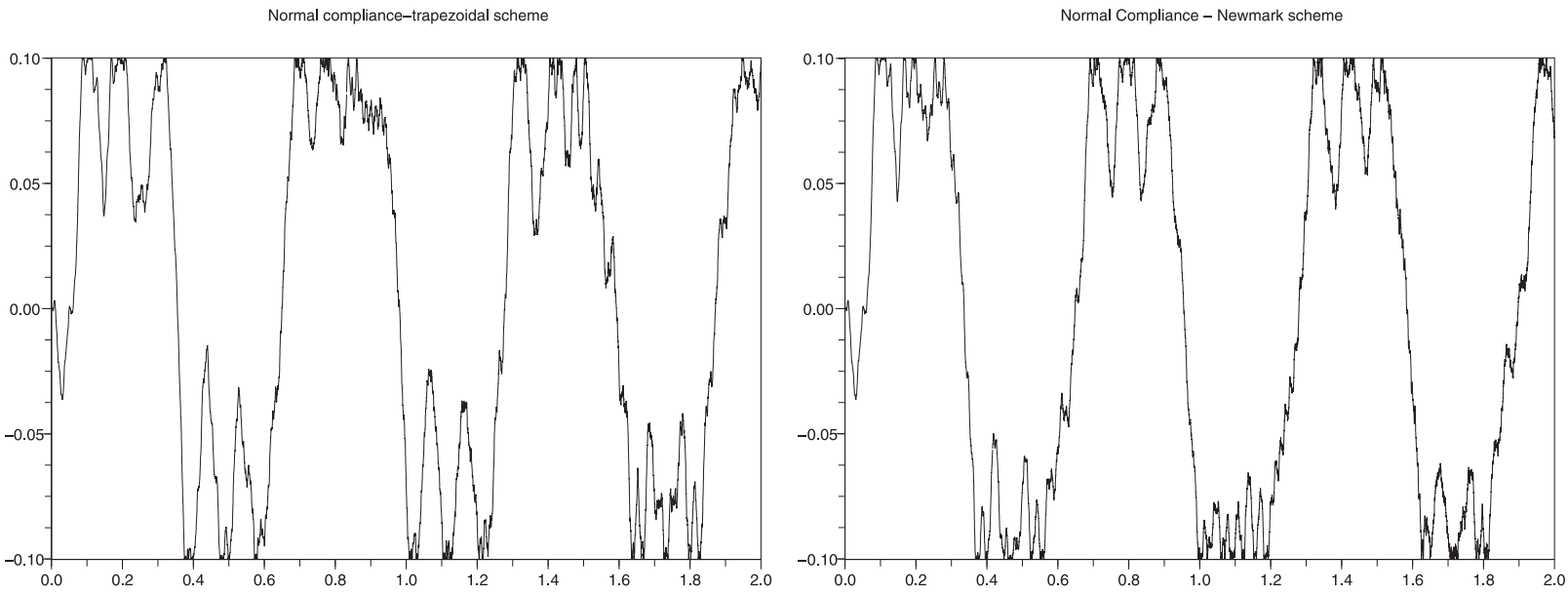

Figure 9. $\Delta t=2 \times 10^{-7} \mathrm{~s}, \beta=\frac{1}{4}$ and $\beta=\frac{1}{2}$.

which are rather coarse approximations of the unilateral constraint. If, we take $1 / \varepsilon=5 \times 10^{9} \mathrm{~N} \cdot \mathrm{m}^{-1}$ with the penalized scheme, we improve the previous result (see Fig. 10), i.e.

$$
\begin{array}{ll}
\max _{0 \leq t \leq 2}\left|u_{\text {app }}(L, t)-g\right|=4.47 \times 10^{-5} \mathrm{~m} & \text { for } \Delta t=2 \times 10^{-7} \mathrm{~s} \text { and } \beta=\frac{1}{4} \\
\max _{0 \leq t \leq 2}\left|u_{\text {app }}(L, t)-g\right|=1.05 \times 10^{-4} \mathrm{~m} & \text { for } \Delta t=2 \times 10^{-7} \mathrm{~s} \text { and } \beta=\frac{1}{2} .
\end{array}
$$

Unfortunately, when $\beta=\frac{1}{2}$ some instabilities appear again after the second contact period (see Fig. 10b).

Finally, for the normal compliance approximation, we display several computations for two "special" values of $\beta$ that are used in structural dynamics: $\beta=\frac{1}{6}$, which corresponds to a linear acceleration, and $\beta=\frac{1}{12}$, which corresponds to the Fox-Goodwin scheme [5]. Even if for linear dynamical systems these two values lead to conditional stability, their periodicity errors are smaller than for $\beta=\frac{1}{4}$, especially for $\beta=\frac{1}{12}$. In Figures 11-13, 

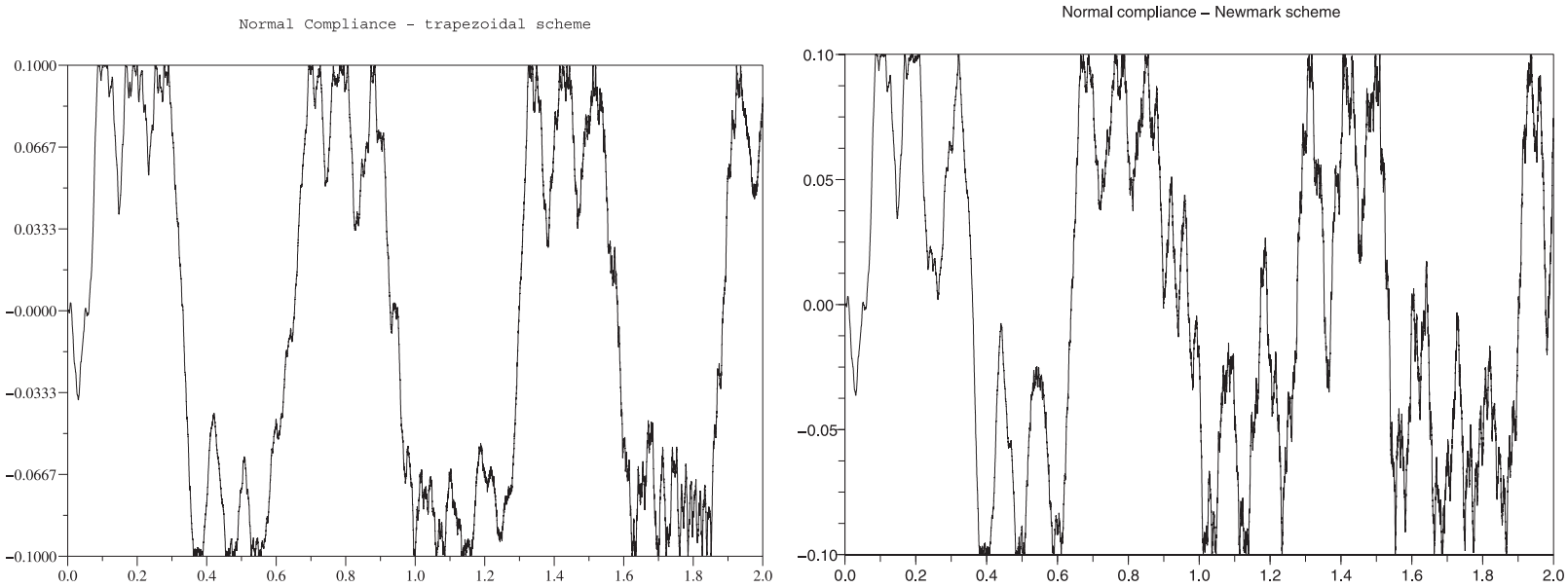

FiguRE 10. $\Delta t=2 \times 10^{-7} \mathrm{~s}, 1 / \varepsilon=5 \times 10^{9}, \beta=\frac{1}{4}$ and $\beta=\frac{1}{2}$.
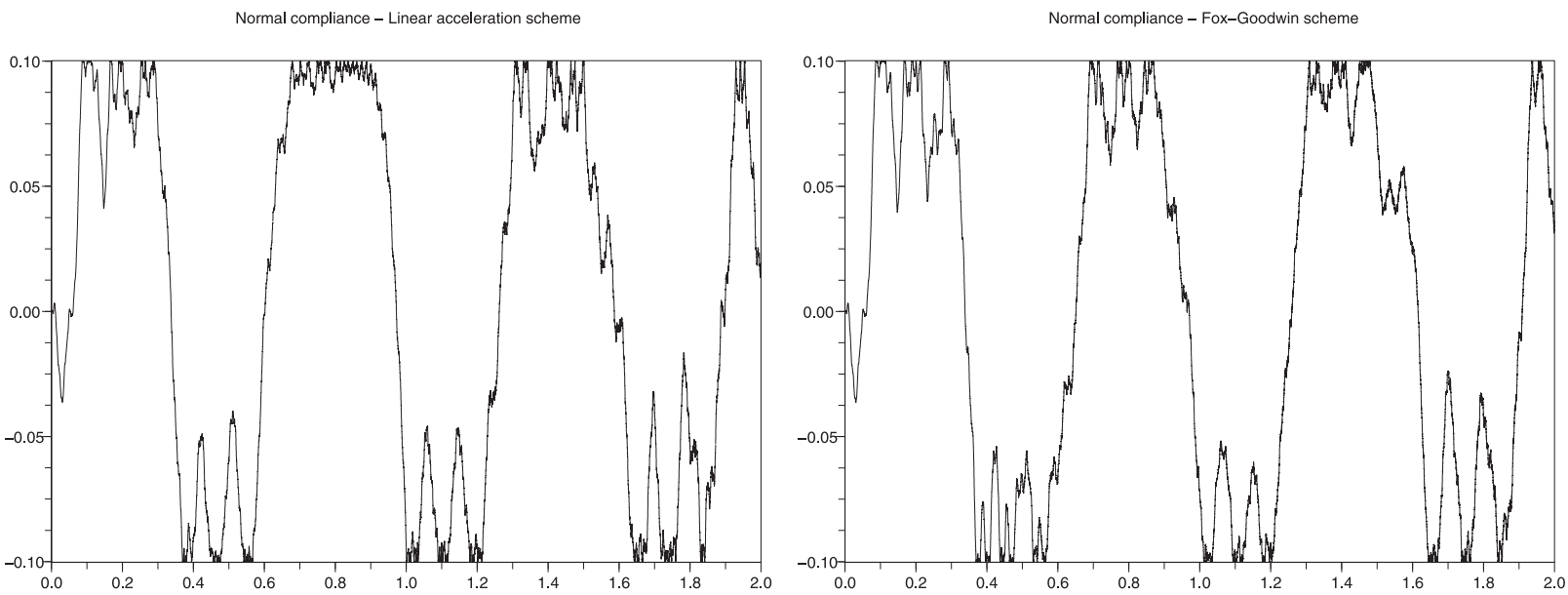

Figure 11. $\Delta t=10^{-6} \mathrm{~s}, \beta=\frac{1}{6}$ and $\beta=\frac{1}{12}$.

we can compare the results obtained with these values of $\beta$. The two schemes perform well (very few numerical instabilities) with comparable CPU-times (see the table at the end of the section).

As expected, the constraint is violated and we get

$$
\begin{array}{ll}
\max _{0 \leq t \leq 2}\left|u_{a p p}(L, t)-g\right|=1.723 \times 10^{-4} \mathrm{~m} & \text { for } \Delta t=2 \times 10^{-7} \mathrm{~s} \text { and } \beta=\frac{1}{12} \\
\max _{0 \leq t \leq 2}\left|u_{\text {app }}(L, t)-g\right|=1.587 \times 10^{-4} \mathrm{~m} & \text { for } \Delta t=2 \times 10^{-7} \mathrm{~s} \text { and } \beta=\frac{1}{6} .
\end{array}
$$



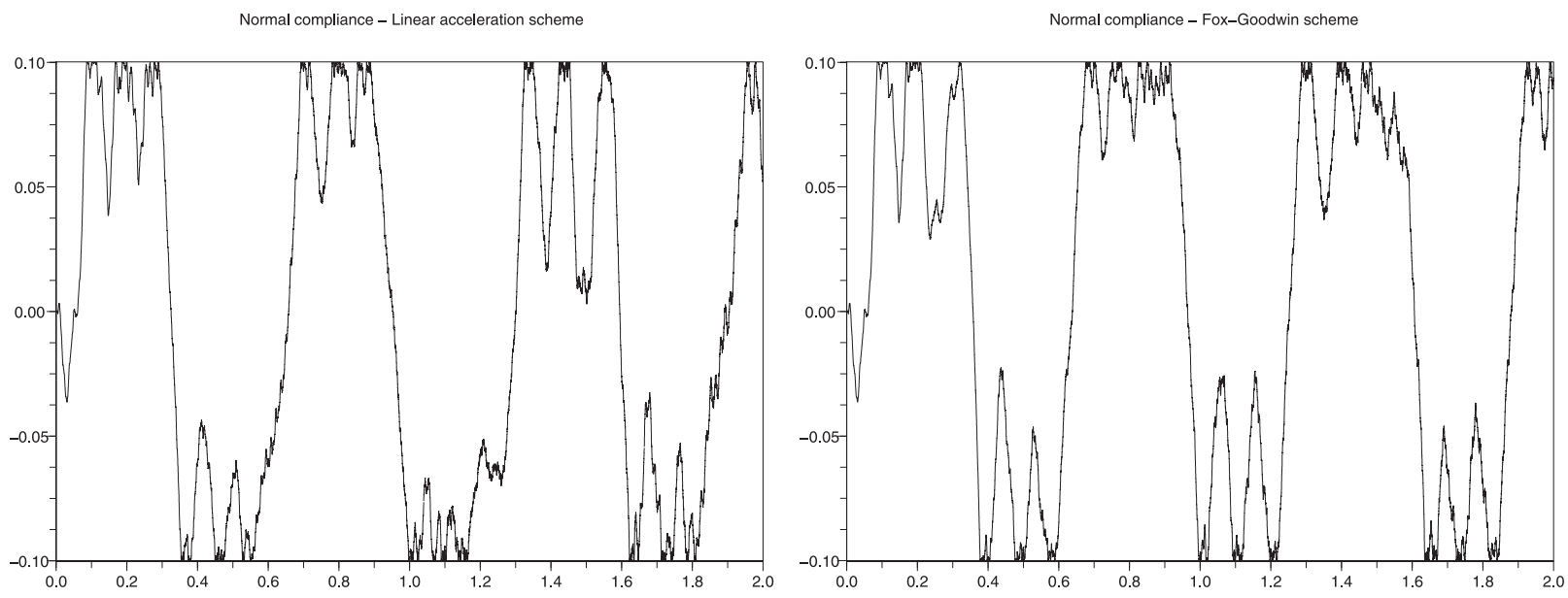

Figure 12. $\Delta t=5 \times 10^{-7} \mathrm{~s}, \beta=\frac{1}{6}$ and $\beta=\frac{1}{12}$.
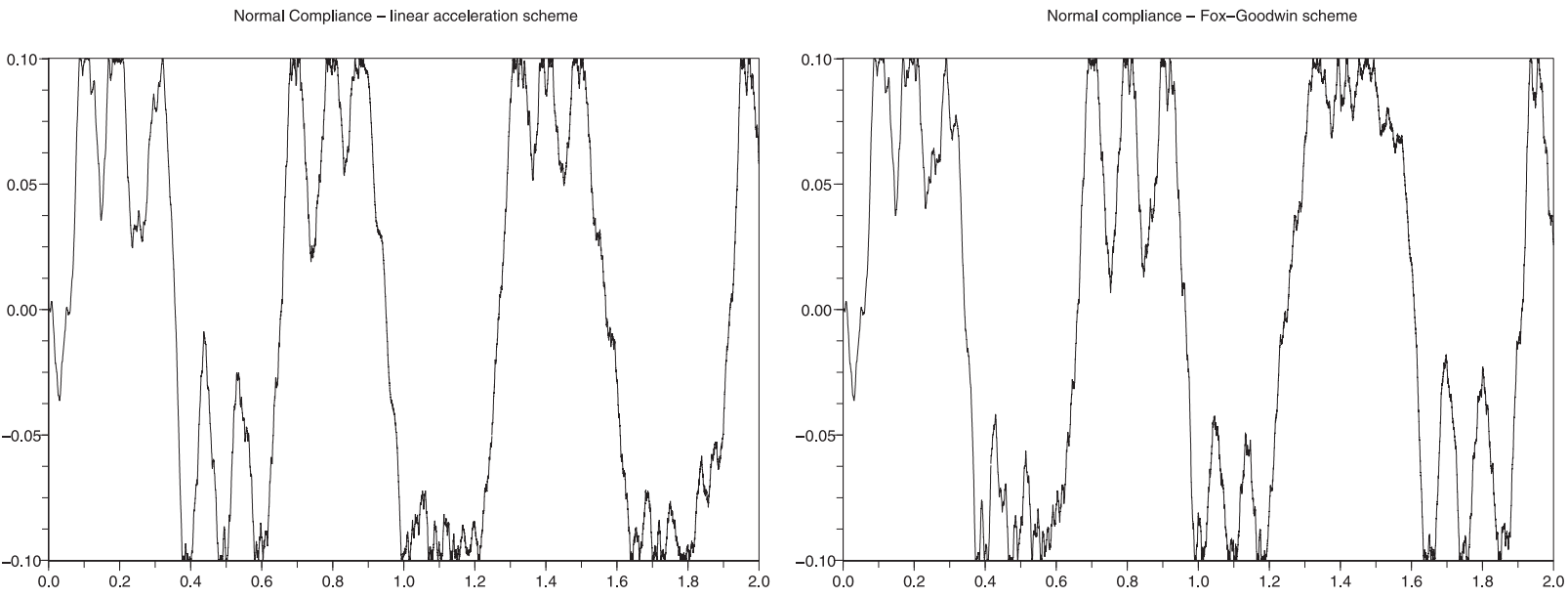

Figure 13. $\Delta t=2 \times 10^{-7} \mathrm{~s}, \beta=\frac{1}{6}$ and $\beta=\frac{1}{12}$.

\begin{tabular}{l|cccccc} 
& Fig. 3a & Fig. 3b & Fig. 4a & Fig. 4b & Fig. 5a & Fig. 5b \\
\hline CPU & & & & & & \\
Time (s) & 15.88 & 151.14 & 753.9 & 747.12 & 1501.51 & 3756.39
\end{tabular}

\begin{tabular}{l|cccccc} 
& Fig. 7a & Fig. 7b & Fig. 8a & Fig. 8b & Fig. 9a & Fig. 9b \\
\hline CPU & & & & & & \\
Time (s) & 1187.9 & 1220.28 & 2426.4 & 2808.28 & 6090 & 6110
\end{tabular}

\begin{tabular}{l|llllll} 
& Fig. 11a & Fig. 11b & Fig. 12a & Fig. 12b & Fig. 13a & Fig. 13b \\
\hline CPU & & & & & & \\
Time (s) & 1214 & 1211.3 & 2431.82 & 2426.13 & 6124.81 & 6066.72
\end{tabular}


Remark 4.2. In the case of the normal compliance condition, we have to compute $\beta \sigma^{n+1}+(1-2 \beta) \sigma^{n}+\beta \sigma^{n-1}$ at each time-step with

$$
\sigma^{n}=-\frac{1}{\varepsilon}\left[\max \left(\bar{u}_{2 J-1}^{n}-g, 0\right)-\max \left(-g-\bar{u}_{2 J-1}^{n}, 0\right)\right] .
$$

Because of the implicit term $\sigma^{n+1}$, we compute an extrapolation of $\bar{u}_{2 J-1}^{n+1}$ in order to obtain an approximation of $\sigma^{n+1}$. This explains why the normal compliance scheme is so expensive in CPU-time in comparison with our scheme.

Remark 4.3. In [4] we consider also other numerical approaches: an event-driven scheme and a time-stepping scheme "à la Moreau". We present several comparisons with the penalty method and the scheme described here which show that our scheme seems the most convenient to solve this type of problems (see [4] for further details).

Remark 4.4. The computations has been performed on MAC G4 (1.1 Ghz) with Scilab 3.1.1, the scientific computing software developed by ENPC-INRIA. Other results are available at the following web address: http://www .univ-reunion.fr/ ydumont/beam-vibrations.htm

\section{Appendix: estimate of $\kappa(h)$.}

We consider the finite element space discretization described at Section 4, i.e. $V_{h}=\operatorname{span}\left\{\varphi_{1}, \ldots, \varphi_{2 J}\right\}$ where $\left(\varphi_{i}\right)_{1 \leq i \leq 2 J}$ is the Hermite piecewise cubics basis. Thus, for all $u_{h} \in V_{h}$ we have

$$
u_{h}=\sum_{i=1}^{J} u_{h}\left(x_{i}\right) \varphi_{2 i-1}+\sum_{i=1}^{J} u_{h}^{\prime}\left(x_{i}\right) \varphi_{2 i} .
$$

In order to simplify the notations, we let

$$
u_{i}=u_{h}\left(x_{i}\right) \quad u_{i}^{\prime}=u_{h}^{\prime}\left(x_{i}\right) \quad \text { for all } i=0, \ldots, J .
$$

We may observe that, since $u_{h} \in V_{h} \subset V$, we have $u_{0}=u_{0}^{\prime}=0$.

Let us compute first $\left(u_{h}, u_{h}\right)$. We have

$$
\begin{aligned}
& \left(u_{h}, u_{h}\right)=\frac{13}{35} \sum_{j=1}^{J}\left(u_{j}^{2}+u_{j-1}^{2}\right) \Delta x+\frac{9}{35} \sum_{j=1}^{J} u_{j} u_{j-1} \Delta x \\
& +\frac{13 \Delta x}{210} \sum_{j=1}^{J}\left(u_{j} u_{j-1}^{\prime}-u_{j-1} u_{j}^{\prime}\right) \Delta x+\frac{(\Delta x)^{2}}{105} \sum_{j=1}^{J}\left(\left(u_{j}^{\prime}\right)^{2}+\left(u_{j-1}^{\prime}\right)^{2}\right) \Delta x \\
& -\frac{(\Delta x)^{2}}{70} \sum_{j=1}^{J} u_{j}^{\prime} u_{j-1}^{\prime} \Delta x-\frac{11(\Delta x)^{2}}{105} u_{J} u_{J}^{\prime} .
\end{aligned}
$$

Then we use the following relations

$$
\begin{aligned}
& \frac{26}{420} \sum_{j=1}^{J} \Delta x\left(u_{j-1} u_{j-1}^{\prime}-u_{j} u_{j}^{\prime}\right) \Delta x=-\frac{26}{420}(\Delta x)^{2} u_{J} u_{J}^{\prime}, \\
& \frac{26}{35} \sum_{j=1}^{J} u_{j}^{2} \Delta x-\frac{13}{35} u_{J}^{2} \Delta x=\frac{13}{35} \sum_{j=1}^{J}\left(u_{j}^{2}+u_{j-1}^{2}\right) \Delta x,
\end{aligned}
$$


and we get

$$
\begin{aligned}
& \left(u_{h}, u_{h}\right)=\frac{1}{140} \sum_{j=1}^{J}\left(\frac{13}{3}\left(u_{j}+u_{j-1}\right)+\Delta x\left(u_{j-1}^{\prime}-u_{j}^{\prime}\right)\right)^{2} \Delta x \\
& +\frac{1752}{7560} \sum_{j=1}^{J-1}\left(u_{j}^{2}+u_{j-1}^{2}\right) \Delta x+\frac{1}{180} \sum_{j=1}^{J}\left(u_{j}-u_{j-1}\right)^{2} \Delta x \\
& +\frac{(\Delta x)^{2}}{420} \sum_{j=1}^{J-1}\left(\left(u_{j}^{\prime}\right)^{2}+\left(u_{j-1}^{\prime}\right)^{2}\right) \Delta x+\frac{(\Delta x)^{3}}{420}\left(u_{J-1}^{\prime}\right)^{2} \\
& +\frac{1}{420}\left(\Delta x u_{J}^{\prime}-9 u_{J}\right)^{2} \Delta x+\frac{1752}{7560} u_{J-1}^{2} \Delta x+\frac{294}{7560} u_{J}^{2} \Delta x .
\end{aligned}
$$

Then, we observe that

$$
\frac{1}{420}\left(\Delta x u_{J}^{\prime}-9 u_{J}\right)^{2} \Delta x+\frac{294}{7560} u_{J}^{2} \Delta x=\frac{1}{420}\left(\frac{18}{19} \Delta x u_{J}^{\prime}-\frac{19}{2} u_{J}\right)^{2} \Delta x+\frac{51}{3024} u_{J}^{2} \Delta x+\frac{37(\Delta x)^{2}}{420 \times 19^{2}}\left(u_{J}^{\prime}\right)^{2} \Delta x
$$

and thus

$$
\begin{aligned}
& \left(u_{h}, u_{h}\right)=\frac{1}{140} \sum_{j=1}^{J}\left(\frac{13}{3}\left(u_{j}+u_{j-1}\right)+\Delta x\left(u_{j-1}^{\prime}-u_{j}^{\prime}\right)\right)^{2} \Delta x \\
& +\frac{1752}{7560} \sum_{j=1}^{J-1}\left(u_{j}^{2}+u_{j-1}^{2}\right) \Delta x+\frac{1752}{7560} u_{J-1}^{2} \Delta x+\frac{1}{180} \sum_{j=1}^{J}\left(u_{j}-u_{j-1}\right)^{2} \Delta x \\
& +\frac{(\Delta x)^{2}}{420} \sum_{j=1}^{J-1}\left(\left(u_{j}^{\prime}\right)^{2}+\left(u_{j-1}^{\prime}\right)^{2}\right) \Delta x+\frac{(\Delta x)^{2}}{420}\left(\left(u_{J-1}^{\prime}\right)^{2}+\frac{37}{19^{2}}\left(u_{J}^{\prime}\right)^{2}\right) \Delta x \\
& +\frac{1}{420}\left(\frac{18}{19} \Delta x u_{J}^{\prime}-\frac{19}{2} u_{J}\right)^{2} \Delta x+\frac{51}{3024} u_{J}^{2} \Delta x
\end{aligned}
$$

We deduce the following inequality

$$
\begin{aligned}
& \left(u_{h}, u_{h}\right) \geq \frac{(\Delta x)^{2}}{420} \frac{37}{19^{2}} \sum_{j=1}^{J}\left(\left(u_{j}^{\prime}\right)^{2}+\left(u_{j-1}^{\prime}\right)^{2}\right) \Delta x+\frac{1}{180} \sum_{j=1}^{J}\left(u_{j}-u_{j-1}\right)^{2} \Delta x \\
& \geq \frac{37}{420 \times 19^{2}}\left((\Delta x)^{2} \sum_{j=1}^{J}\left(\left(u_{j}^{\prime}\right)^{2}+\left(u_{j-1}^{\prime}\right)^{2}\right) \Delta x+\sum_{j=1}^{J}\left(u_{j}-u_{j-1}\right)^{2} \Delta x\right) .
\end{aligned}
$$

Now we compute $a\left(u_{h}, u_{h}\right)$ :

$$
\begin{aligned}
& a\left(u_{h}, u_{h}\right)=\frac{k^{2}}{(\Delta x)^{4}}\left(12 \sum_{j=1}^{J}\left(u_{j}^{2}+u_{j-1}^{2}\right) \Delta x-24 \sum_{j=1}^{J} u_{j} u_{j-1} \Delta x\right) \\
& +\frac{k^{2}}{(\Delta x)^{4}}\left(12 \Delta x \sum_{j=1}^{J}\left(u_{j-1} u_{j}^{\prime}-u_{j} u_{j-1}^{\prime}\right) \Delta x+4(\Delta x)^{2} \sum_{j=1}^{J} u_{j}^{\prime} u_{j-1}^{\prime} \Delta x\right) \\
& +\frac{k^{2}}{(\Delta x)^{4}}\left(4(\Delta x)^{2} \sum_{j=1}^{J}\left(\left(u_{j}^{\prime}\right)^{2}+\left(u_{j-1}^{\prime}\right)^{2}\right) \Delta x-12(\Delta x)^{2} u_{J} u_{J}^{\prime}\right),
\end{aligned}
$$


which gives

$$
a\left(u_{h}, u_{h}\right)=\frac{k^{2}}{(\Delta x)^{4}}\left(3 \sum_{j=1}^{J}\left(2\left(u_{j}-u_{j-1}\right)-\Delta x\left(u_{j}^{\prime}+u_{j-1}^{\prime}\right)\right)^{2} \Delta x+\left(\Delta x^{2}\right) \sum_{j=1}^{J}\left(u_{j}^{\prime}-u_{j-1}^{\prime}\right)^{2} \Delta x\right) .
$$

Hence, using the classical inequality

$$
a b \leq \frac{a^{2}}{4 \varepsilon}+\varepsilon b^{2} \quad \forall(a, b) \in \mathbb{R}^{2}, \quad \forall \varepsilon>0,
$$

we get

$$
\begin{aligned}
& a\left(u_{h}, u_{h}\right) \leq \frac{k^{2}}{(\Delta x)^{4}}\left(3\left(4+\frac{1}{\varepsilon}\right) \sum_{j=1}^{J}\left(u_{j}-u_{j-1}\right)^{2} \Delta x\right. \\
& \left.+3(1+4 \varepsilon)\left(\Delta x^{2}\right) \sum_{j=1}^{J}\left(u_{j}^{\prime}+u_{j-1}^{\prime}\right)^{2} \Delta x+\left(\Delta x^{2}\right) \sum_{j=1}^{J}\left(u_{j}^{\prime}-u_{j-1}^{\prime}\right)^{2} \Delta x\right) \\
& \leq \max \left(3\left(4+\frac{1}{\varepsilon}\right), 6(1+4 \varepsilon)\right) \frac{k^{2}}{(\Delta x)^{4}} \\
& \times\left(\sum_{j=1}^{J}\left(u_{j}-u_{j-1}\right)^{2} \Delta x+\left(\Delta x^{2}\right) \sum_{j=1}^{J}\left(\left(u_{j}^{\prime}\right)^{2}+\left(u_{j-1}^{\prime}\right)^{2}\right) \Delta x\right) .
\end{aligned}
$$

With the choice $\varepsilon=1 / 2$, we deduce

$$
a\left(u_{h}, u_{h}\right) \leq \frac{18 k^{2}}{(\Delta x)^{4}} \frac{420 \times 19^{2}}{37}\left(u_{h}, u_{h}\right), \quad \forall u_{h} \in V_{h}
$$

and thus

$$
\kappa(h) \leq \tilde{\kappa}(h)=\frac{18 \times 420 \times 19^{2}}{37} \frac{k^{2}}{(\Delta x)^{4}}=\frac{2^{3} \times 5 \times 19^{2} \times 7 \times 3^{3}}{37} \frac{k^{2}}{(\Delta x)^{4}} .
$$

Remark 5.1. This is certainly not an optimal upper bound for $\kappa(h)$.

\section{REFERENCES}

[1] B. Brogliato, A.A. ten Dam, L. Paoli, F. Genot and M. Abadie, Numerical simulation of finite dimensional multibody nonsmooth mechanical systems. ASME Appl. Mechanics Rev. 55 (2002) 107-149.

[2] Y. Dumont, Vibrations of a beam between stops: Numerical simulations and comparison of several numerical schemes. Math. Comput. Simul. 60 (2002) 45-83.

[3] Y. Dumont, Some remarks on a vibro-impact scheme. Numer. Algorithms 33 (2003) 227-240.

[4] Y. Dumont and L. Paoli, Simulations of beam vibrations between stops: comparison of several numerical approaches, in Proceedings of the Fifth EUROMECH Nonlinear Dynamics Conference (ENOC-2005), CD Rom (2005).

[5] L. Fox, The numerical solution of two-point boudary values problems in ordinary differential equations, Oxford University Press, New York (1957).

[6] J. Guckenheimer and P. Holmes, Nonlinear oscillations, dynamical systems and bifurcations of vector fields. Springer-Verlag, New York, Berlin, Heidelberg (1983).

[7] T. Hughes, The finite element method. Linear static and dynamic finite element analysis. Prentice-Hall International, Englewood Cliffs (1987).

[8] K. Kuttler and M. Shillor, Vibrations of a beam between two stops. Dynamics of continuous, discrete and impulsive systems, Series B, Applications and Algorithms 8 (2001) 93-110. 
[9] C.H. Lamarque and O. Janin, Comparison of several numerical methods for mechanical systems with impacts. Int. J. Num. Meth. Eng. 51 (2001) 1101-1132.

[10] F.C. Moon and S.W. Shaw, Chaotic vibration of a beam with nonlinear boundary conditions. Int. J. Nonlinear Mech. 18 (1983) 465-477.

[11] L. Paoli, Analyse numérique de vibrations avec contraintes unilatérales. Ph.D. thesis, University of Lyon 1, France (1993).

[12] L. Paoli, Time-discretization of vibro-impact. Phil. Trans. Royal Soc. London A. 359 (2001) $2405-2428$.

[13] L. Paoli, An existence result for non-smooth vibro-impact problems. Math. Mod. Meth. Appl. S. (M3AS) 15 (2005) 53-93.

[14] L. Paoli and M. Schatzman, Mouvement à un nombre fini de degrés de liberté avec contraintes unilatérales : cas avec perte d'énergie. RAIRO Modél. Math. Anal. Numér. 27 (1993) 673-717.

[15] L. Paoli and M. Schatzman, Ill-posedness in vibro-impact and its numerical consequences, in Proceedings of European Congress on COmputational Methods in Applied Sciences and engineering (ECCOMAS), CD Rom (2000).

[16] L. Paoli and M. Schatzman, A numerical scheme for impact problems, I and II. SIAM Numer. Anal. 40 (2002) 702-733; $734-768$.

[17] P. Ravn, A continuous analysis method for planar multibody systems with joint clearance. Multibody Syst. Dynam. 2 (1998) $1-24$.

[18] R.T. Rockafellar, Convex analysis. Princeton University Press, Princeton (1970).

[19] M. Schatzman and M. Bercovier, Numerical approximation of a wave equation with unilateral constraints. Math. Comp. 53 (1989) 55-79.

[20] S.W. Shaw and R.H. Rand, The transition to chaos in a simple mechanical system. Int. J. Nonlinear Mech. 24 (1989) 41-56.

[21] J. Simon, Compact sets in the space $L^{p}(0, T ; B)$. Ann. Mat. Pur. Appl. 146 (1987) 65-96.

[22] D. Stoianovici and Y. Hurmuzlu, A critical study of applicability of rigid body collision theory. ASME J. Appl. Mech. 63 (1996) 307-316.

To access this journal online:

www.edpsciences.org 